\title{
LAS NUEVAS FORMAS DE LA METRÓPOLIS Tejidos metropolitanos, ríos y cuencas, flujos de movilidad, reservas y multiescalaridad en las metrópolis latinoamericanas contemporáneas

\author{
THE NEW FORMS OF THE METROPOLIS \\ Metropolitan fabrics, rivers and basins, urban reserves, and multiscalarity in \\ contemporary Latin American metropolises
}

Jaimes, César;

(Instituto Superior de Urbanismo, Territorio y Ambiente, Universidad de Buenos Aires)

pjaimesarq@fadu.uba.ar

Amette, Roberto

(Instituto Superior de Urbanismo, Territorio y Ambiente, Universidad de Buenos Aires)

roberto.amette@fadu.uba.ar

\section{RESUMEN}

La presente investigación apunta al estudio y a la producción de nuevas versiones de forma urbana metropolitana en escalas precisas de particular relevancia, tomando como caso a la Región Metropolitana de Buenos Aires en comparación con otros casos latinoamericanos. El trabajo tiene eje en dos objetivos que se retroalimentan: en primer lugar, la producción de un cuerpo de conocimiento que permita su transferencia en el ámbito académico, y en segundo lugar, la identificación de pautas fundamentales de generación y transformación de la forma urbana que, siendo sistémicas en la dinámica de urbanización de la Región Metropolitana de Buenos Aires, puedan nutrir el diseño de políticas públicas en la materia a través de la producción de instrumentos de actuación específicos. La presente ponencia presenta parte de los resultados del primer año de investigación.

Palabras clave: Urbanismo, Forma, Metrópolis

Bloque temático Morfologías urbanas

\begin{abstract}
This project points to both research and produce new versions of metropolitan urban form in particularly relevant scales, focusing on Buenos Aires Metropolitan Region in comparison with other Latin American metropolitan areas. The work rests on two goals that feedback each other: first, the production of a body of knowledge transferable to academic environments, and in a second place, the identification of certain fundamental blueprints for generating and transforming urban form, systemic to the urbanization dynamics in Buenos Aires Metropolitan Region and thus capable of nurturing the design of specific public policies through the production of specific intervention instruments. This paper advances some of the results produced during the first year of the research.
\end{abstract}

Keywords: Urbanism, Form, Metropolis

Topic: Urban morphologies 


\section{Introducción}

La presente investigación, que se desarrolla durante los años 2018 y 2019, apunta al estudio y a la producción de nuevas versiones de forma urbana metropolitana en escalas precisas de particular relevancia, tomando como caso a la Región Metropolitana de Buenos Aires (RMBA) en comparación con otros casos latinoamericanos.

El trabajo tiene eje en dos objetivos que se retroalimentan: en primer lugar, la producción de un cuerpo de conocimiento que permita su transferencia en el ámbito académico, y en segundo lugar, la identificación de pautas fundamentales de generación y transformación de la forma urbana que, siendo sistémicas en la dinámica de urbanización de la Región Metropolitana de Buenos Aires, puedan nutrir el diseño de políticas públicas en la materia a través de la producción de instrumentos de actuación específicos.

En este sentido, cabe considerar que la propuesta de investigación se realizó en 2017 en forma contemporánea a la creación, a fines de 2016 y en el ámbito del Ministerio del Interior, Obras Públicas y Vivienda del Gobierno Nacional de la República Argentina, de la Comisión Consultiva del Área Metropolitana de Buenos Aires (CO.C.A.M.B.A.), instancia que "tendrá a su cargo elaborar propuestas de innovación institucional para el área en cuestión, que se sustenten en el desarrollo humano, económico, tecnológico, ambiental y urbano, para la región metropolitana" (Considerandos al Decreto 1126/2016 de creación de la CO.C.A.M.B.A., 26/10/2016). La Región Metropolitana de Buenos Aires carece a la fecha de un organismo que aborde integralmente su gestión, la que implica a jurisdicciones en tres niveles del Estado argentino: federal (la Ciudad Autónoma de Buenos Aires -CABA- es sede del Gobierno Nacional, siendo el Río de la Plata asimismo de jurisdicción nacional), provincial (la Provincia de Buenos Aires -PBA-, y la misma Ciudad Autónoma de Buenos Aires, que tiene rango provincial) y municipal (la RMBA comprende a la CABA y a los 40 municipios que la circundan, todos pertenecientes a la PBA). La creación de la CO.C.A.M.B.A., junto a otros organismos ya existentes y en proceso de constitución que abordan aspectos importantes de la dimensión metropolitana, al mismo tiempo que existen Programas Interdisciplinarios de Investigación de la Universidad de Buenos Aires alineados con este objeto de estudio, hacen oportuno un abordaje multiescalar como el que propone la presente investigación. Este abordaje tiene como objetivo reconocer e incidir en los nuevos procesos policéntricos urbanos, promoviendo nuevos instrumentos de planeamiento transjurisdiccionales que proporcionen esquemas de desarrollo integral, equilibrado y evolutivo, considerando al mismo tiempo las relaciones recíprocas de las fuerzas naturales y culturales en las regiones metropolitanas y las relaciones internas de estas fuerzas en el espacio de cada ciudad.

La necesidad de contar con estudios y propuestas de actuación integrales y sectoriales actualizados acerca del desenvolvimiento de las formas urbanas metropolitanas latinoamericanas, que incluyan sus relaciones con ríos y cuencas, flujos de movilidad, y reservas urbanas y naturales, y la coincidencia de la presente investigación con los mencionados trabajos en el ámbito gubernamental y académico, han supuesto la oportunidad de construir en adelante una colaboración interinstitucional auspiciosa. Ello es particularmente importante en el marco institucional en el que se encuadra esta investigación: los Proyectos de Desarrollo Estratégico de Investigación de la Universidad, que aspiran a generar resultados que puedan ser transferidos de manera directa a actores adoptantes, actores que los propios proyectos deben identificar y que aceptan recibir tales resultados.

En nuestro caso, tales actores adoptantes son:

1. El Gobierno de la Ciudad de Buenos Aires, a través de su Secretaría General y de Relaciones Internacionales, entre cuyos objetivos se encuentra la "... coordinación y seguimiento de iniciativas interjurisdiccionales para el desarrollo y la implementación de políticas metropolitanas" (carta de colaboración y adopción de resultados, Gobierno de la Ciudad de Buenos Aires, 2018).

2. La Corporación Buenos Aires Sur Sociedad del Estado, cuyo objetivo es “... contribuir al desarrollo social, económico y urbanístico de todo el Sur de la Ciudad Autónoma de Buenos Aires" (carta de colaboración y adopción de resultados, Corporación Buenos Aires Sur Sociedad del Estado, 2017). 


\section{Hipótesis, objetivos y metodología}

El proyecto parte de la hipótesis de que si bien es evidente la existencia de dinámicas urbanas generalizadas que responden a patrones históricos y contemporáneos de asentamiento y edificación para las actividades residenciales, industriales, comerciales y de servicios en las grandes ciudades y metrópolis, no existe un conocimiento sistemático y suficientemente preciso de las dimensiones escalares de su alcance ni de su magnitud en términos de consumo de suelo, de interacción con los soportes naturales, de relación con infraestructuras metropolitanas existentes y por construir, y de favorecimiento de adecuadas y estables condiciones espaciales para el trabajo, la salud, la vida social, la actividad económica y los derechos humanos de las poblaciones.

En este marco, el objetivo de la investigación es indagar acerca de ciertas escalas físicas de estudio y actuación que puedan verificarse como las más apropiadas, por sus características de inteligibilidad y posibilidad operativa, para operar diacrónicamente en los tejidos metropolitanos en su consistencia interna y en su relación con ríos y cuencas, flujos de movilidad, y áreas de reserva urbanas y naturales.

A tal efecto, metodológicamente, las escalas de estudio y actuación para la Región Metropolitana de Buenos Aires que postula el proyecto se disponen en un orden multiescalar de 10 rangos que, en la hipótesis de partida, puede ser segmentado, de mayor a menor, en ordenamientos territoriales-metropolitanos (de 40.000 a $1.500 \mathrm{~km} 2$ de área de suelo), ordenamientos urbanos (de 300 a $12 \mathrm{~km} 2$ de área de suelo), unidades de diseño urbano (de 250 a $10 \mathrm{Ha}$ de área de suelo), y unidades de arquitectura (de $2 \mathrm{Ha}$ a la dimensión del pequeño lote urbano). Para cada una de estas 4 escalas de trabajo, es posible definir rangos internos, totalizando 10 (Tabla 1, Figuras 4 a 8 ).

En la etapa final del segundo año de investigación, se definirán y pondrán a prueba instrumentos de interpretación e intervención que, operando de manera ejemplar en ciertas escalas precisas de ordenamiento y de unidades de diseño urbanos (provenientes de los rangos definidos), sean capaces de incidir positivamente en las escalas de ordenamiento territorial-metropolitano y de ordenamiento urbano.

En cuanto a sus resultados, al cabo de sus dos años de duración el proyecto propone:

1. Producir un Atlas de Instrumentos Urbanísticos, tanto preexistentes como propuestos por el proyecto mismo.

2. Determinar un conjunto acotado de ordenamientos y unidades de diseño urbanos susceptibles de promover como política pública, desde el gobierno nacional, provincial, local o metropolitano, según las problemáticas características detectadas y el orden escalar definido.

Un emergente clave de la presente investigación será la comprobación de la relevancia de las escalas físicas metropolitanas estudiadas para la producción de instrumentos urbanísticos (planes, programas y proyectos de intervención y regulación) capaces de ser transferidos a la práctica de gestión de los actores institucionales adoptantes de los resultados.

En el contexto descripto, la etapa de investigación desarrollada durante 2018, motivo de la presente ponencia, ha propuesto en primer lugar, luego de la construcción de su corpus documental y su puesta a punto metodológica, estudiar diacrónicamente, por una parte, los instrumentos urbanísticos producidos por diferentes organismos del Estado argentino a partir de 1880, fecha en la cual se designa a Buenos Aires ciudad capital de la República Argentina; y por otra, compilar instrumentos urbanísticos producidos por la propia Universidad, en el ámbito de la enseñanza de grado de arquitectura y urbanismo. En segundo término, ha producido una primera caracterización escalar de cinco tipos de instrumentos urbanísticos estudiados. 


\section{La Región Metropolitana de Buenos Aires}

Los acontecimientos centrales para el ingreso de Buenos Aires en la condición metropolitana fueron los hechos que determinaron, entre 1880 y 1882, dos decisiones de naturaleza política: su establecimiento como Capital Federal de la República Argentina (Ley Nacional de 1880), y la fundación de la ciudad de La Plata como nueva Capital de la Provincia de Buenos Aires (Ley Provincial de 1882), culminando décadas de disputa, desde 1816, por dar al nuevo país una forma de organización definitiva y establecer su ciudad capital. Es así como la definición del nuevo límite de la ciudad de Buenos Aires, y el trazado del ejido de la nueva Ciudad de La Plata, establecieron los instrumentos urbanísticos fundantes de la edad metropolitana de Buenos Aires; y fueron luego seguidos por numerosos otros, desde fines del siglo XIX y a lo largo de los siglos XX y XXI, estableciendo una cultura urbanística propia.

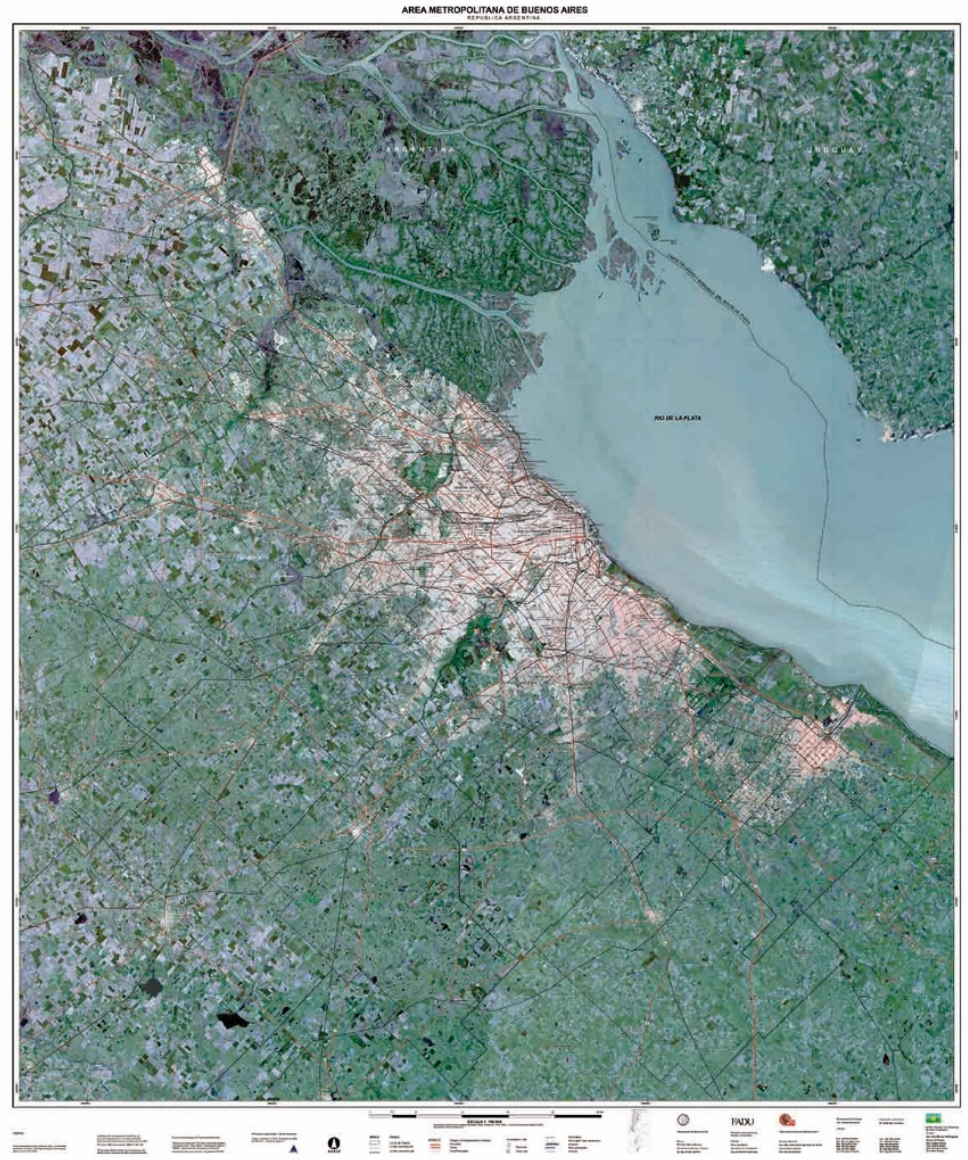

Figura 1. Región Metropolitana de Buenos Aires, imagen satelital-territorial. Fuente: Centro de Información Metropolitana, Instituto Superior de Urbanismo, Territorio y Ambiente, Universidad de Buenos Aires (2017)

La dimensión, escala y condición de los variados instrumentos urbanísticos que se propusieron y, en muchos casos, se llevaron a cabo o aún operan (regulaciones, proyectos urbanos, planes urbanos, organismos y programas), fueron y son atributos muy importantes en los 140 años que median entre 1880 y 2020 , y continuarán siéndolo en el futuro. En este período, las leyes, el diseño y el planeamiento urbanos se entendieron de maneras distintas, aun en el marco de un mismo paradigma general (el del desarrollo de una ciudad capitalista occidental latinoamericana): desde las unidades de hasta $200 \mathrm{~km} 2$ que definían un recinto urbano gobernable dotado de áreas de expansión interna (en la Ley Nacional de 1880), pasando por los ordenamientos territoriales-metropolitanos de $25.000 \mathrm{~km} 2$ que aspiraban a planificar y dar servicios a toda una metrópolis en expansión (década de 1960), los instrumentos de "acupuntura urbana" de diseño de 1 ó 2km2, 
capaces de regenerar áreas intraurbanas degradadas (operaciones de la década de 1990), hasta los renovados y actuales estudios de organización del área/región metropolitana (2007-presente).

Hoy, acercándonos a 2020, asistimos a una transición en el modo de entender qué instrumentos urbanísticos pueden ser los más idóneos para dar ciertas referencias de orden y regeneración urbanos a metrópolis que, en el marco de las cambiantes o inciertas organizaciones posindustriales, ya no son susceptibles de ser planificadas completamente (Díaz, 2013). En este contexto, es clave renovar y resignificar una colaboración estratégica entre Universidad y Organismos de gobierno para que, a través de la investigación científica y el cuestionamiento creativo de las prácticas vigentes, puedan identificarse nuevas pautas fundamentales de generación y transformación de la forma urbana, capaces de nutrir el diseño de políticas públicas en la materia, a través de la producción de instrumentos de actuación específicos.

\section{Situación actual de la Región Metropolitana de Buenos Aires}

La Región se extiende a lo largo de la costa sudoccidental del estuario del Río de la Plata, dentro de un arco semicircular de unos $75 \mathrm{~km}$ de radio, con centro en el sitio de la segunda fundación de la ciudad por Juan de Garay en 1580. Lo hace sobre una llanura de pampa ondulada de escasa pendiente, comprendiendo dos cuencas hidrográficas relevantes y altamente urbanizadas (Riachuelo-Matanza y Río de la Reconquista), otra escasamente urbanizada (Río Luján), y numerosas cuencas menores. Habitan en la RMBA, según el Censo Nacional 2010, casi 15 millones de personas.

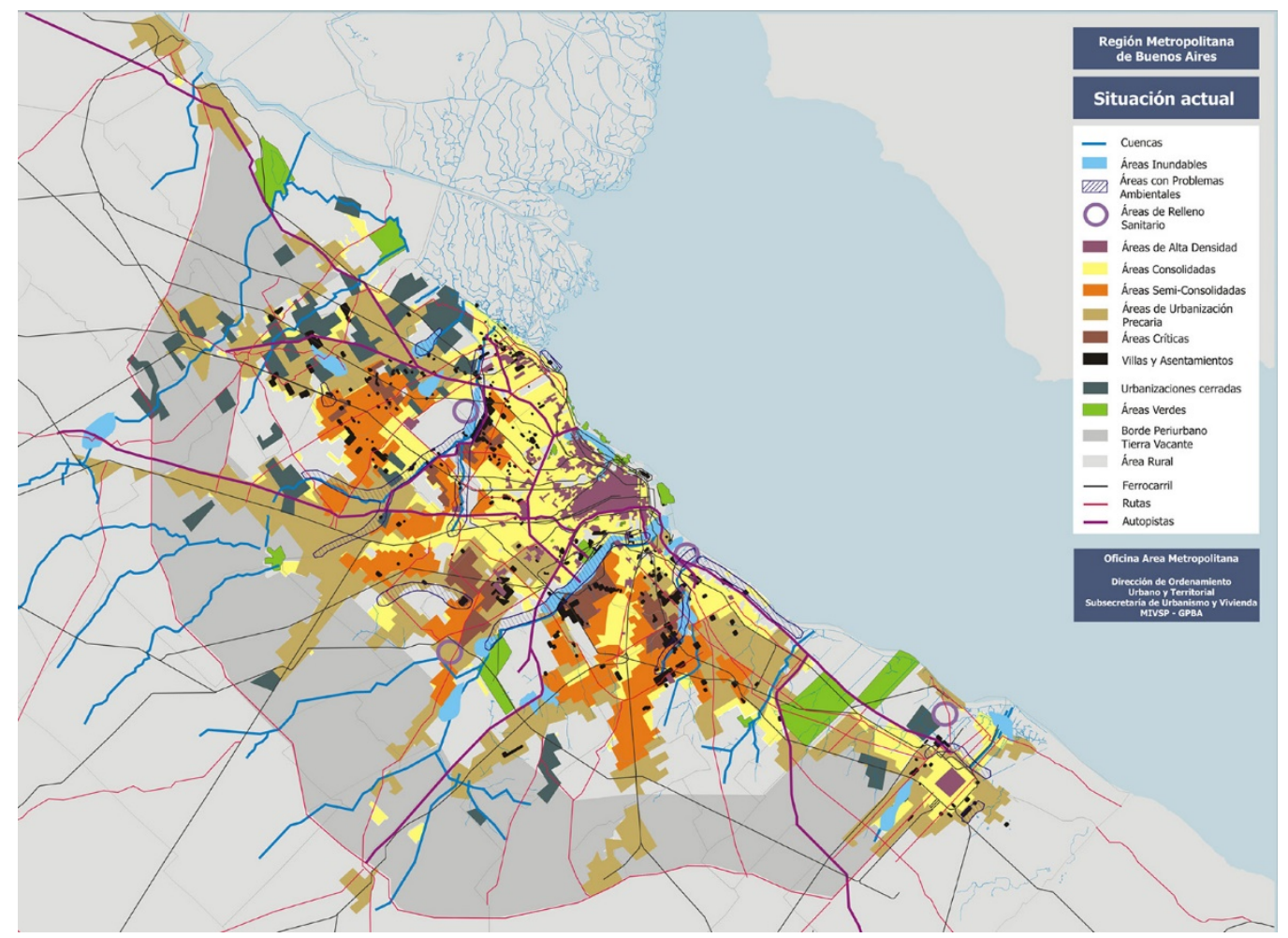

Figura 2. Región Metropolitana de Buenos Aires. Situación actual. Fuente: Plan Estratégico Territorial. Subsecretaría de Planificación Territorial de la Inversión Pública, Ministerio de Planificación Federal, Inversión Pública y Servicios (2008)

La Ciudad de Buenos Aires con su área comarcal, de escasa importancia hasta fines del Siglo XVIII durante la dominación española, es designada en 1776 capital del Virreinato del Río de la Plata. Su población protagoniza los hechos que, a partir de 1810, conducirían a la emancipación del país. La organización definitiva de la Argentina culmina con el establecimiento de Buenos Aires como ciudad capital y su federalización (1880), y la contemporánea creación de la ciudad de La Plata, $60 \mathrm{~km}$ al Sudeste, como nueva capital de la Provincia de 
Buenos Aires (1882). La incorporación del país al nuevo escenario económico internacional de mediados a fines del Siglo XIX imprime a la ciudad de Buenos Aires una rápida transformación y extensión, a través de la construcción de grandes obras de infraestructura de transporte (puerto, ferrocarriles) y de servicios (principalmente de saneamiento), y de una activa política inmigratoria.

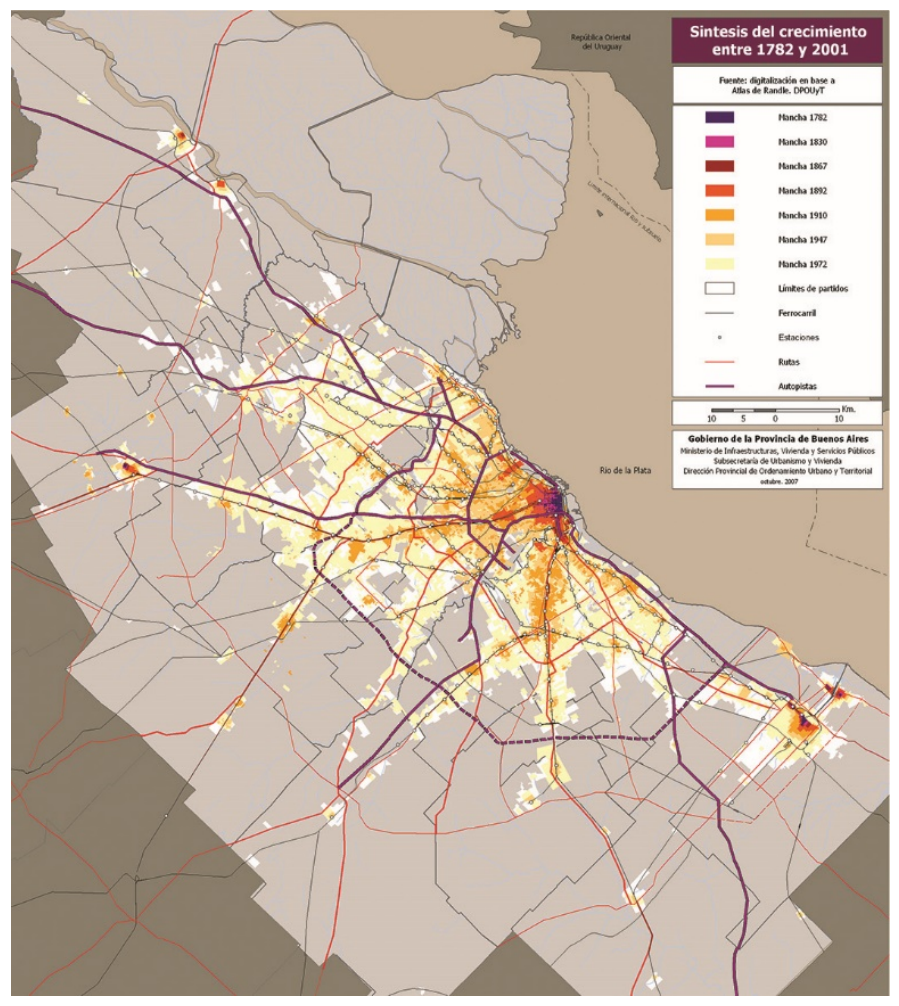

Figura 3. Región Metropolitana de Buenos Aires. Síntesis de crecimiento. Fuente: Lineamientos Estratégicos para la Región Metropolitana de Buenos Aires. Dirección Provincial de Ordenamiento Urbano y Territorial, Subsecretaría de Urbanismo y Vivienda, Ministerio de Infraestructura, Vivienda y Servicios Públicos, Gobierno de la Provincia de Buenos Aires (2006)

El crecimiento metropolitano se fue estructurando de manera tentacular, a través de cinco ejes de tendidos ferroviarios que, a partir de la década de 1850, se disponen sobre la previa red caminera virreinal. Estos tendidos promueven una temprana suburbanización que, a partir de la década de 1940, se intensifica con el desarrollo industrial derivado de la sustitución de importaciones durante la II Guerra Mundial. A partir de la década de 1930, con el desarrollo del automóvil y el transporte automotor, comienzan las inversiones públicas camineras, que a partir de 1960 incluyen el tendido de autopistas. Hasta la década de 1990, prácticamente la totalidad de la urbanización se desarrolla según un modelo abierto de grilla derivado de la estructura fundacional de Juan de Garay. Desde la década de 1990, prevalece un modelo de crecimiento y de acceso al suelo fragmentado, basado en la creación de urbanizaciones cerradas para sectores medios y altos, y en el crecimiento de villas y asentamientos para quienes no pueden acceder al suelo y a la vivienda por vías formalizadas.

La Región Metropolitana de Buenos Aires presenta una marcada gradiente de densidades, desde un máximo de unos 150Hab/Ha en la Ciudad Autónoma y unos 60-100Hab/Ha en los distritos de su conurbación inmediata, hasta los 10-40Hab/Ha del segundo cordón de conurbación y densidades inferiores a los $5 \mathrm{Hab} / \mathrm{Ha}$ en los distritos del periurbano, eminentemente rurales. 


\section{Las Nuevas Formas de la Metrópolis. Etapa 1 de la Investigación: Primera Fase}

"En consecuencia, volvemos a encontrarnos con el antiguo problema (pero como se ve, también actual) que es el de darle "forma" a la ciudad, a lo urbano. Para poder controlar el crecimiento de lo urbano-ciudad y de sus partes o áreas de influencia, es necesario limitarlas, definirlas, en resumen, darles "forma". Por supuesto que el concepto contemporáneo de "forma" de lo urbano no tiene nada que ver con el de la ciudad tradicional o de aquellos modelos de la historia del urbanismo. La escala y los problemas actuales hacen de la cuestión de la "forma" un problema bien distinto que el suscitado en épocas anteriores. Pero esta es una de las cuestiones que hay que plantearse y resolver y vale tanto a escala del territorio como de lo urbano construido. Si se trata de poner límites a la ocupación del suelo, límites significa "forma", implica a la política en su sentido más general de soberanía y poder, y de ello hay que preocuparse." (Díaz, 2013: 185)

Denominadas Caracterización y jerarquización de las escalas físicas e instrumentos de actuación que puedan verificarse como las más apropiadas para operar en los tejidos urbanos del sistema de la Región Metropolitana de Buenos Aires, las tareas de la Etapa 1 del proyecto han estado orientadas principalmente a construir una robusta plataforma cartográfica, acompañada por una guía metodológica y teórica que, además de servir a los propios objetivos de la investigación, proporcionen un marco adecuado para la formación de los docentesinvestigadores que integran el proyecto, y de los estudiantes de grado que realizan sus pasantías de formación en investigación; sentando de este modo las bases para, en el segundo año, producir nuevos instrumentos urbanísticos en este marco, y proporcionar líneas para la continuidad futura del trabajo.

\subsection{Puesta a punto metodológica. Construcción de corpus documental.}

\subsubsection{Revisión y pautado preciso del orden escalar de estudio.}

El proyecto parte de un orden escalar-cartográfico orientado a proporcionar, en un número de rangos acotado, la posibilidad de estudiar lo metropolitano desde la escala mayor de lo territorial -donde se inscribe la metrópolis entera-, hasta la escala de la arquitectura, unidad urbana mínima. Para ello, se organiza un orden fijo de representación, en el que a la división por 2 de la escala cartográfica, se corresponde un área enmarcada en una carta de dimensión A0 de la cuarta parte de la superficie del rango inmediato anterior.

\begin{tabular}{|c|c|c|c|c|c|c|c|}
\hline \multirow{2}{*}{\multicolumn{2}{|c|}{ Escala 1: }} & & \multicolumn{5}{|c|}{ Representación A0 (X=1180mm x Y=840mm) } \\
\hline & & Instrumento & ${ }^{\star} \mathrm{Ha}$ total & ${ }^{\star} \mathrm{km} 2$ total & km2 total & $X(\mathbf{k m})$ & $\mathbf{Y ( k m )}$ \\
\hline 1 & 200.000 & Ordenamiento Territorial & 4.000 .000 & 40.000 & 39.648 & 236,00 & 168,00 \\
\hline 2 & 100.000 & Ordenamiento Metropolitano 1 & 1.000 .000 & 10.000 & 9.912 & 118,00 & 84,00 \\
\hline 3 & 50.000 & Ordenamiento Metropolitano 2 & 250.000 & 2.500 & 2.478 & 59,00 & 42,00 \\
\hline 4 & 25.000 & Ordenamiento Urbano 1 & 62.500 & 625 & 620 & 29,50 & 21,00 \\
\hline $5-$ & 15.000 & \multirow{3}{*}{ Ordenamiento Urbano 2} & 22.500 & 225 & 223 & 17,70 & 12,60 \\
\hline 5 & 12.500 & & 15.000 & 150 & 155 & 14,75 & 10,50 \\
\hline $5+$ & 10.000 & & 10.000 & 100 & 99 & 11,80 & 8,40 \\
\hline 6 & 5.000 & Ordenamiento Urbano 3 & 2.500 & 25 & 25 & 5,90 & 4,20 \\
\hline 7 & 2.500 & Proyecto Urbano 1 & 600 & \begin{tabular}{ll|}
6,00 \\
\end{tabular} & 6,20 & 2,95 & 2,10 \\
\hline 8 & 1.000 & Proyecto Urbano 2 & 100 & 1,00 & 0,99 & 1,18 & 0,84 \\
\hline 9 & 500 & Proyecto Urbano 3 & 25 & 0,25 & 0,25 & 0,59 & 0,42 \\
\hline 10 & 250 & Arquitectura & 6 & 0,06 & 0,06 & 0,30 & 0,21 \\
\hline
\end{tabular}

Tabla 1. Orden escalar de estudio. Fuente: Proyecto de Desarrollo Estratégico (PDE) Las Nuevas Formas de la Metrópolis, Universidad de Buenos Aires / Instituto Superior de Urbanismo, Territorio y Ambiente (2018)

De este modo, quedan estructuradas la identificación y revisión del tamaño de las unidades territoriales, metropolitanas y urbanas a estudiar; las escalas de su representación cartográfica; y la magnitud primaria de los instrumentos urbanísticos que propenden a su ordenamiento físico, funcional y significativo. 


\subsubsection{Compilación de la información cartográfica disponible.}

Para el caso de la Región Metropolitana de Buenos Aires, es ostensible la carencia de una fuente compilada que integre, de modo operable y consistente, la información cartográfica disponible: componentes topográficos e hidrográficos, estructura de movilidad, división distrital, orden parcelario, tejidos urbanos, reservas urbanas y naturales. Fue necesario, por lo tanto, abordar esta etapa de compilación, que estuvo basada en la integración de fuentes diferentes y su ordenamiento en una única y coherente base digital y de comunicación.

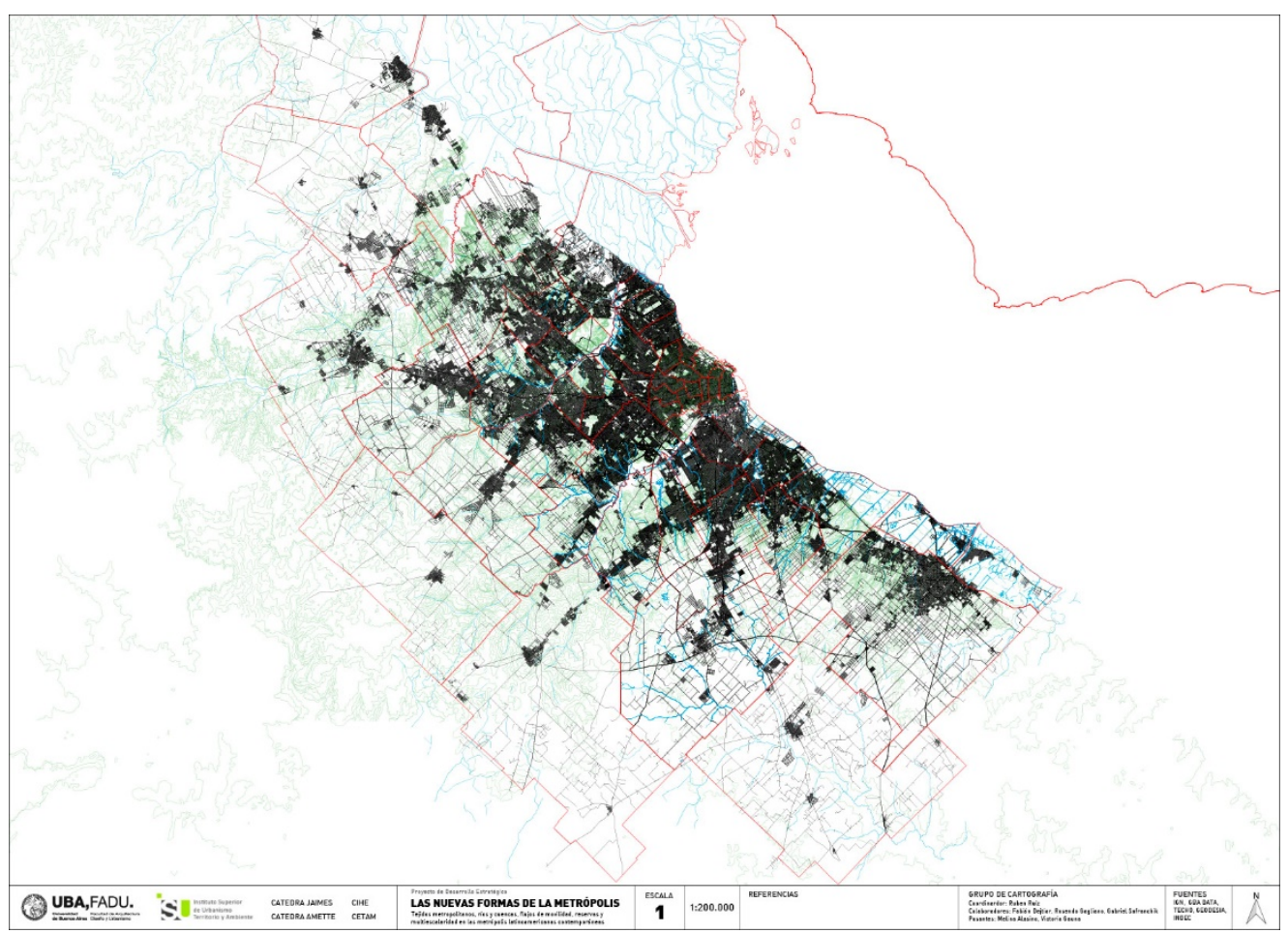

Figura 4. Región Metropolitana de Buenos Aires. Compilación de información cartográfica: componentes topográficos e hidrográficos estructura de movilidad, división distrital, orden parcelario, tejidos urbanos, reservas urbanas y naturales. Escala 1 (1:200.000). Área territorial de $40.000 \mathrm{~km}^{2}$. Fuente: Proyecto de Desarrollo Estratégico (PDE) Las Nuevas Formas de la Metrópolis, Universidad de Buenos Aires / Instituto Superior de Urbanismo, Territorio y Ambiente (2018) 


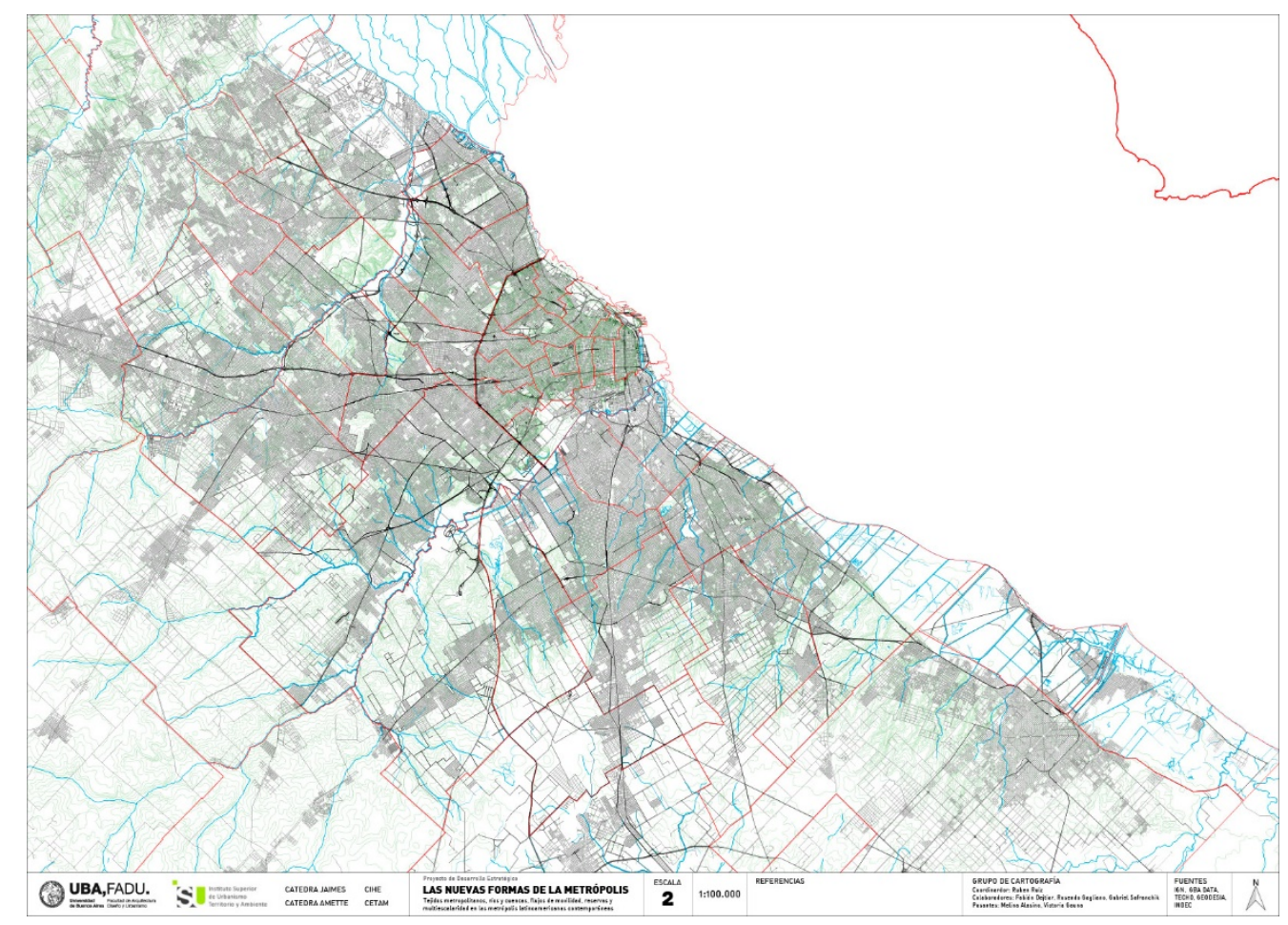

Figura 5. Región Metropolitana de Buenos Aires. Compilación de información cartográfica: componentes topográficos e hidrográficos, estructura de movilidad, división distrital, orden parcelario, tejidos urbanos, reservas urbanas y naturales. Escala 2 (1:100.000). Área metropolitana de $10.000 \mathrm{~km}^{2}$. Fuente: Proyecto de Desarrollo Estratégico (PDE) Las Nuevas Formas de la Metrópolis, Universidad de Buenos Aires / Instituto Superior de Urbanismo, Territorio y Ambiente (2018)

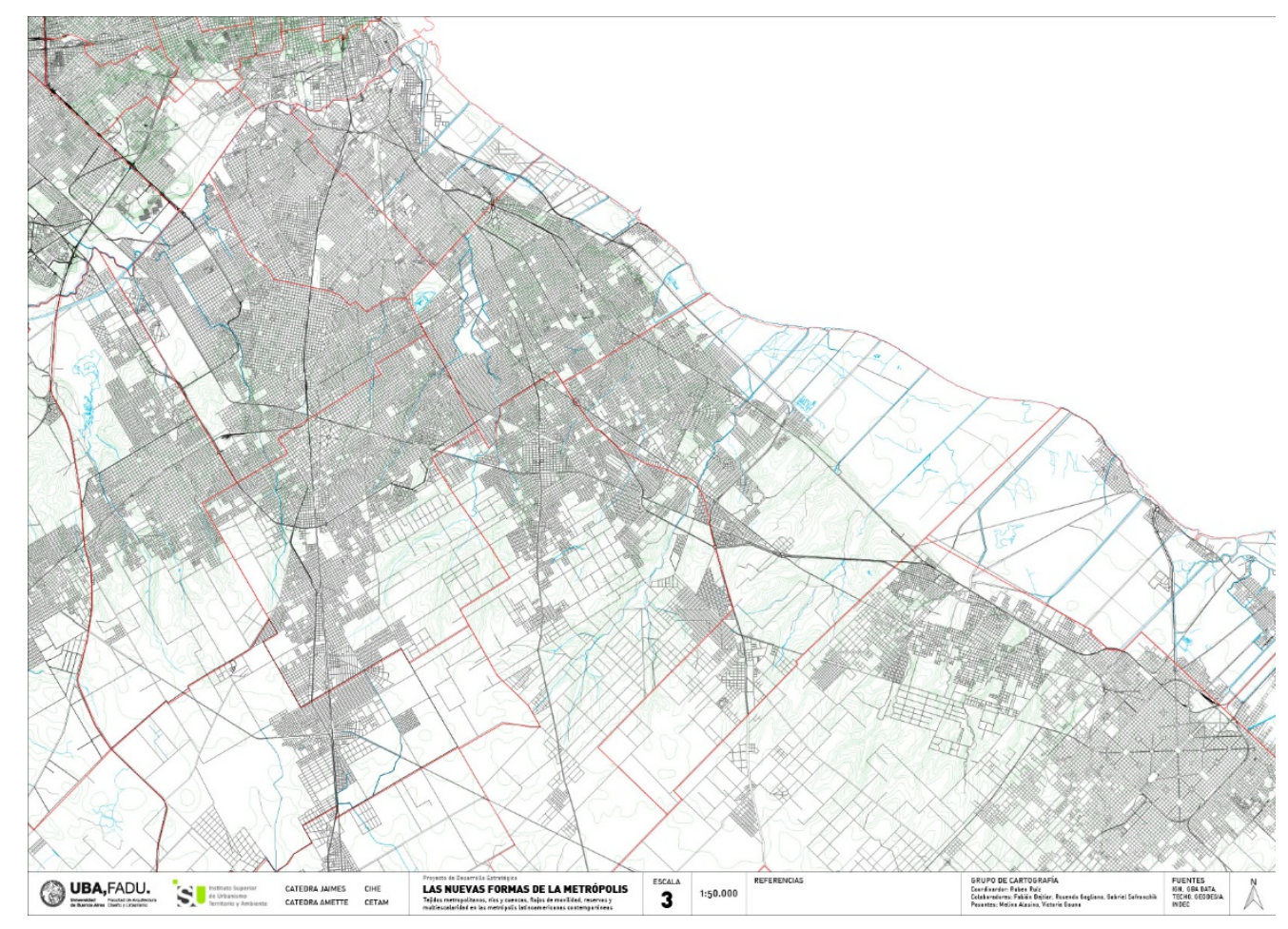

Figura 6. Región Metropolitana de Buenos Aires. Compilación de información cartográfica: componentes topográficos e hidrográficos, estructura de movilidad, división distrital, orden parcelario, tejidos urbanos, reservas urbanas y naturales. Escala 3 (1:50.000). Área metropolitana de $2.500 \mathrm{~km}^{2}$. Fuente: Proyecto de Desarrollo Estratégico (PDE) Las Nuevas Formas de la Metrópolis, Universidad de Buenos Aires / Instituto Superior de Urbanismo, Territorio y Ambiente (2018) 


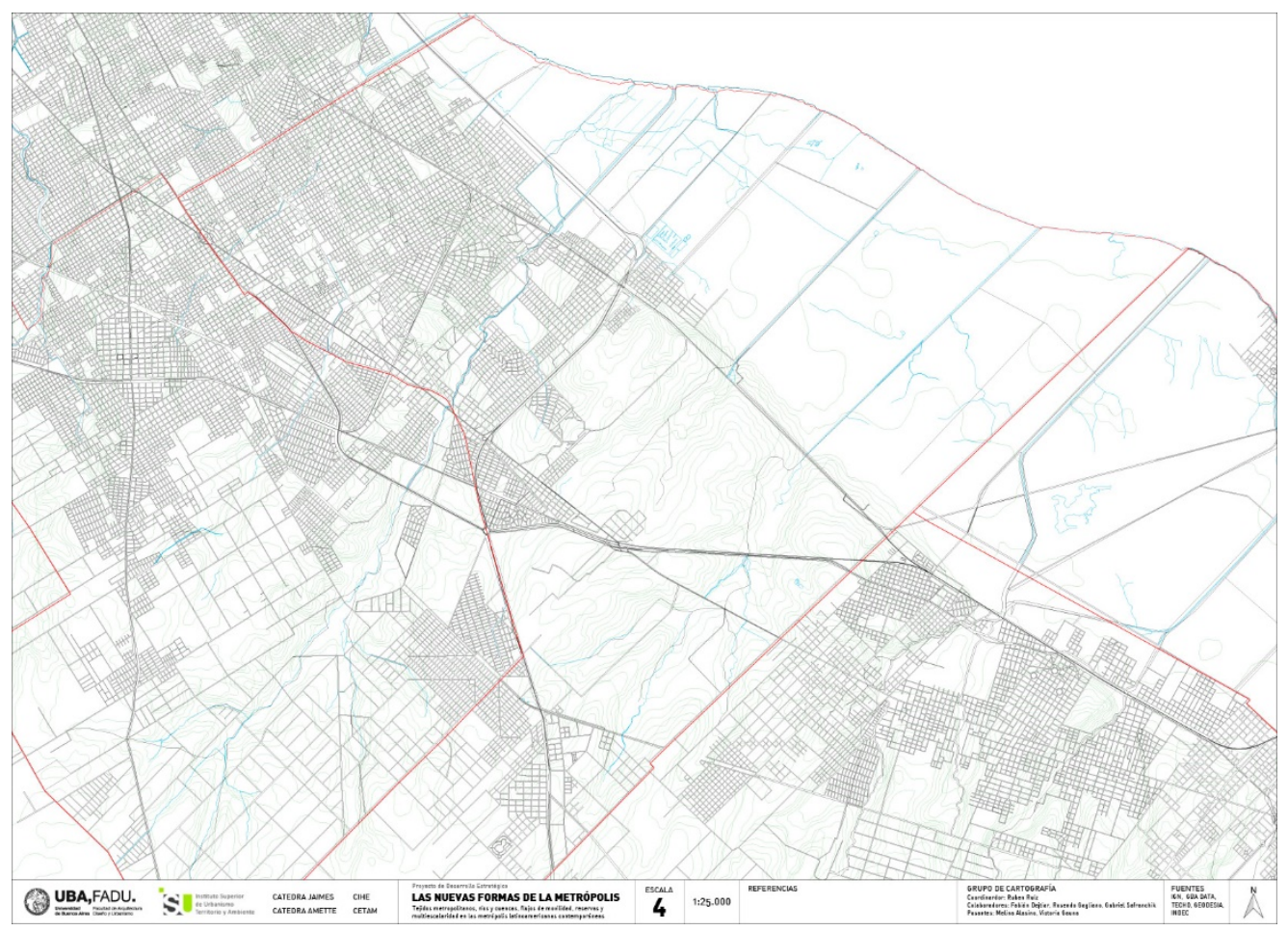

Figura 7. Región Metropolitana de Buenos Aires. Compilación de información cartográfica: componentes topográficos e hidrográficos, estructura de movilidad, división distrital, orden parcelario, tejidos urbanos, reservas urbanas y naturales. Escala 4 (1:25.000). Área urbana de $625 \mathrm{~km}^{2}$. Fuente: Proyecto de Desarrollo Estratégico (PDE) Las Nuevas Formas de la Metrópolis, Universidad de Buenos Aires / Instituto Superior de Urbanismo, Territorio y Ambiente (2018)

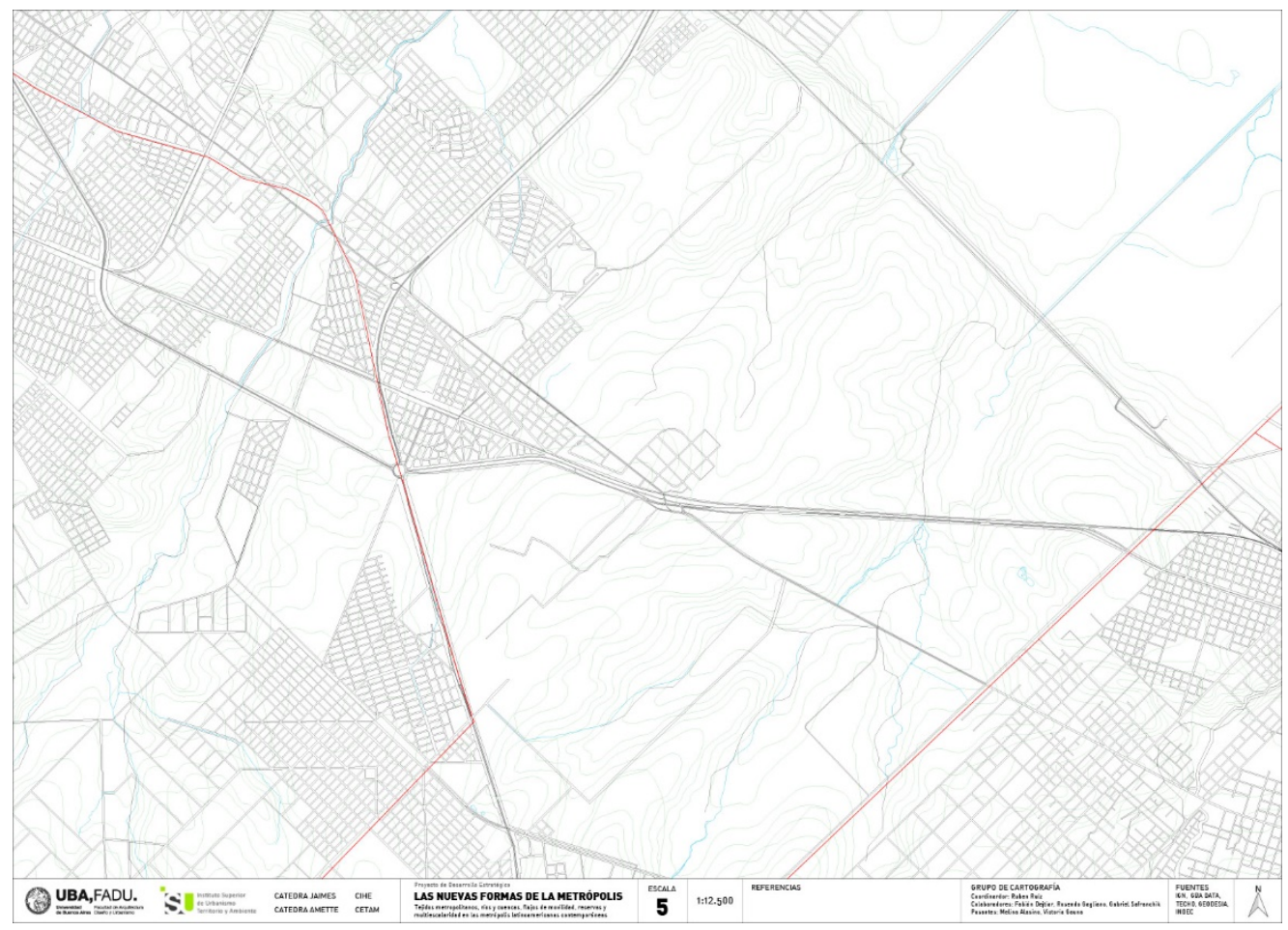

Figura 8. Región Metropolitana de Buenos Aires. Compilación de información cartográfica: componentes topográficos e hidrográficos, estructura de movilidad, división distrital, orden parcelario, tejidos urbanos, reservas urbanas y naturales. Escala 5 (1:12.500). Área urbana de $150 \mathrm{~km}^{2}$. Fuente: Proyecto de Desarrollo Estratégico (PDE) Las Nuevas Formas de la Metrópolis, Universidad de Buenos Aires / Instituto Superior de Urbanismo, Territorio y Ambiente (2018) 


\subsection{Recopilación de planes, proyectos, programas y regulaciones, 1880-2020.}

4.2.1. Recopilación de planes, proyectos, programas y regulaciones para las diferentes jurisdicciones involucradas (nacional, provincial, municipal e interjurisdiccional). Definición de la periodización del material recopilado.

El objetivo del proyecto respecto a producir nuevos instrumentos urbanísticos no puede partir sino del reconocimiento y el estudio de aquellos que han sido producidos en la historia. Para el caso de la Región Metropolitana de Buenos Aires, ellos han sido muy numerosos, parten desde el momento mismo de la capitalización de la ciudad (con antecedentes relevantes en el período virreinal y posterior a la Independencia), y tienen siempre como constante la transformación o postulación de alternativas a su grilla fundacional, patrón de su crecimiento y consolidación hasta la década de 1990.

El primer año de trabajo ha compilado unos cincuenta instrumentos históricos y actuales, cuyas siluetas se han redibujado y transferido a la cartografía en el orden escalar definido.

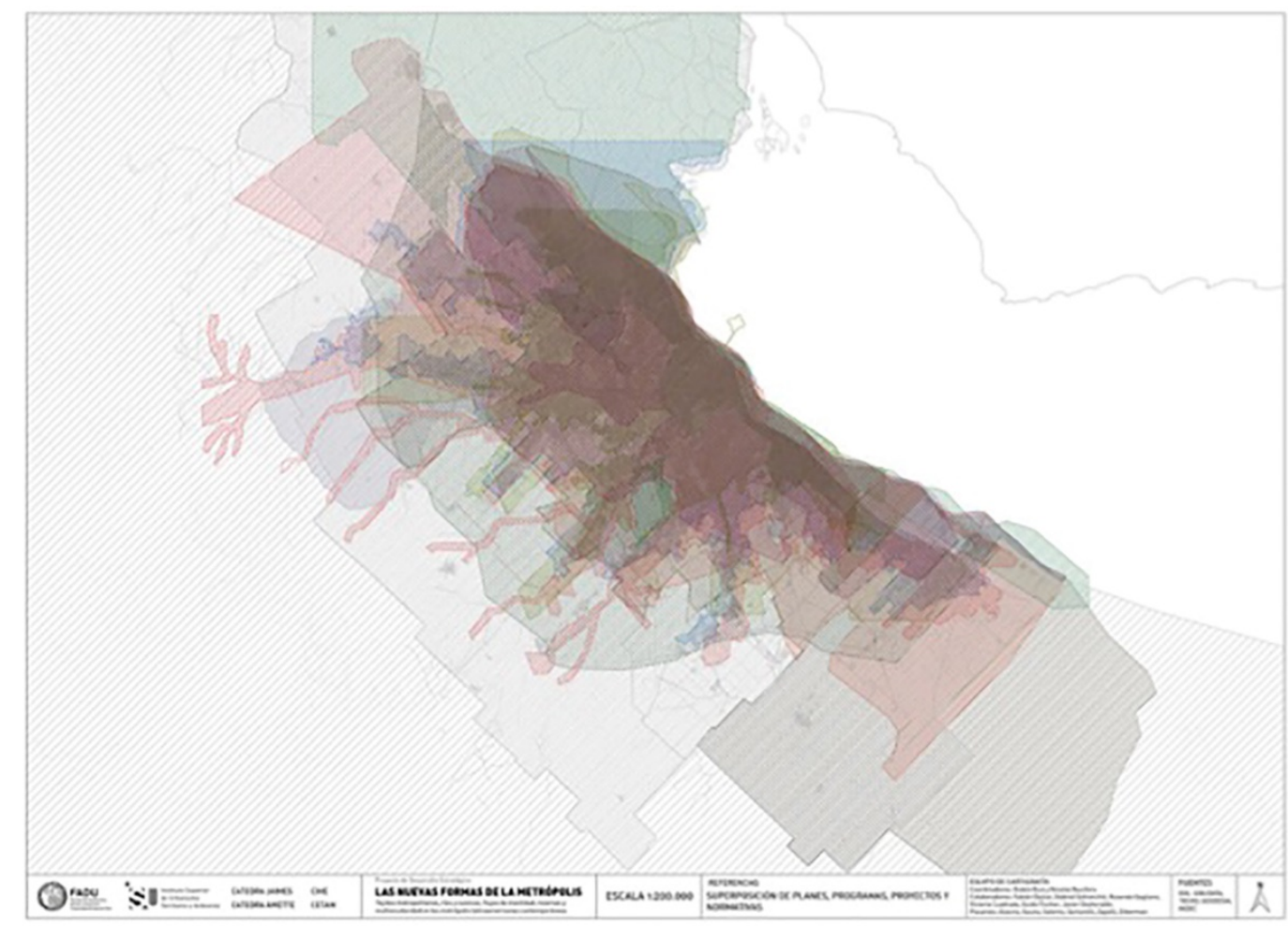

Figura 9. Región Metropolitana de Buenos Aires. Superposición de instrumentos urbanísticos metropolitanos entre 1880 y 2020 . Escala 1 (1:200.000). Área territorial de 40.000 $\mathrm{km}^{2}$. Fuente: Proyecto de Desarrollo Estratégico (PDE) Las Nuevas Formas de la Metrópolis, Universidad de Buenos Aires / Instituto Superior de Urbanismo, Territorio y Ambiente (2018) 


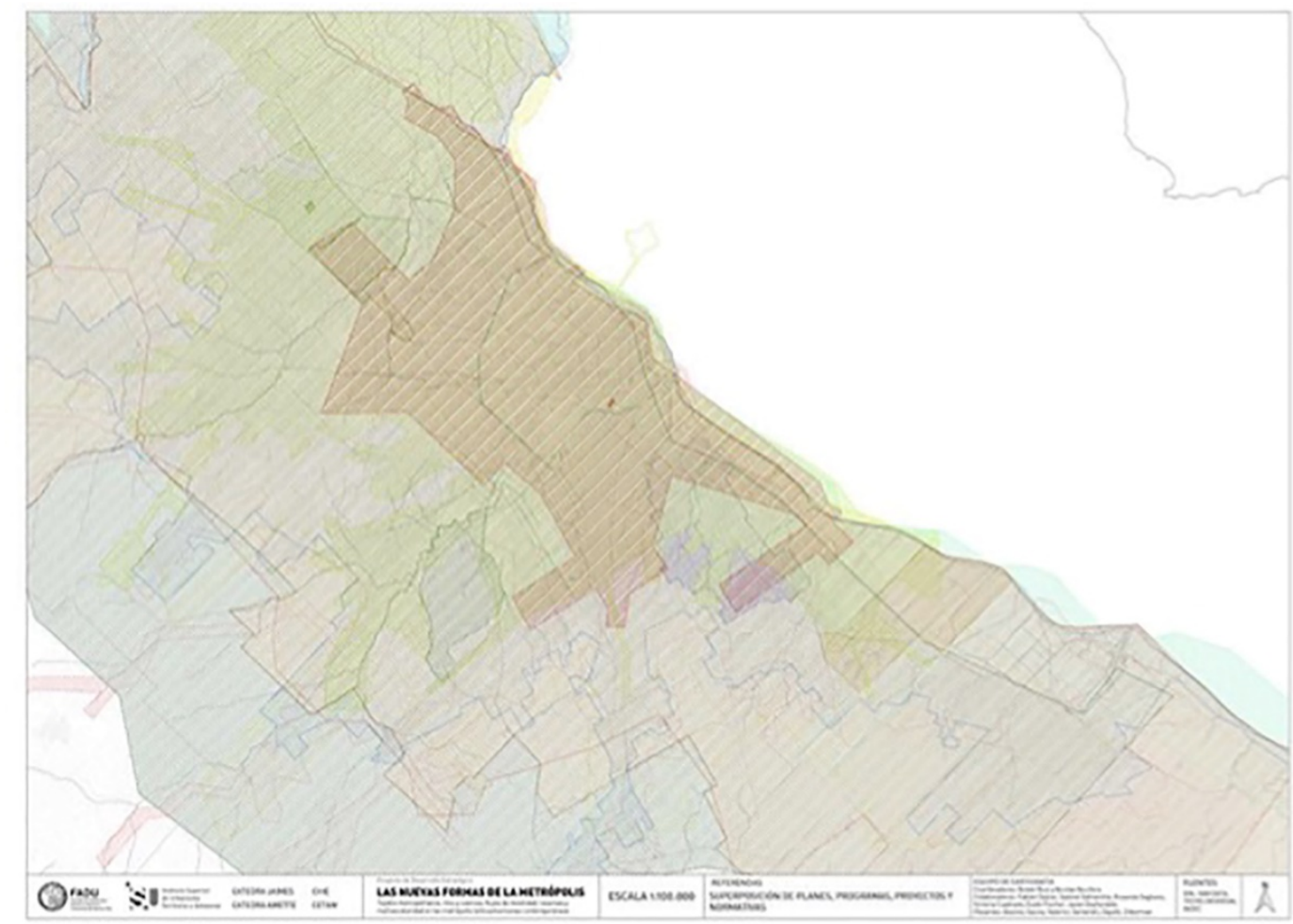

Figura 10. Región Metropolitana de Buenos Aires. Superposición de instrumentos urbanísticos metropolitanos entre 1880 y 2020 . Escala 2 (1:100.000). Área metropolitana de 10.000km². Fuente: Proyecto de Desarrollo Estratégico (PDE) Las Nuevas Formas de la Metrópolis, Universidad de Buenos Aires / Instituto Superior de Urbanismo, Territorio y Ambiente (2018)

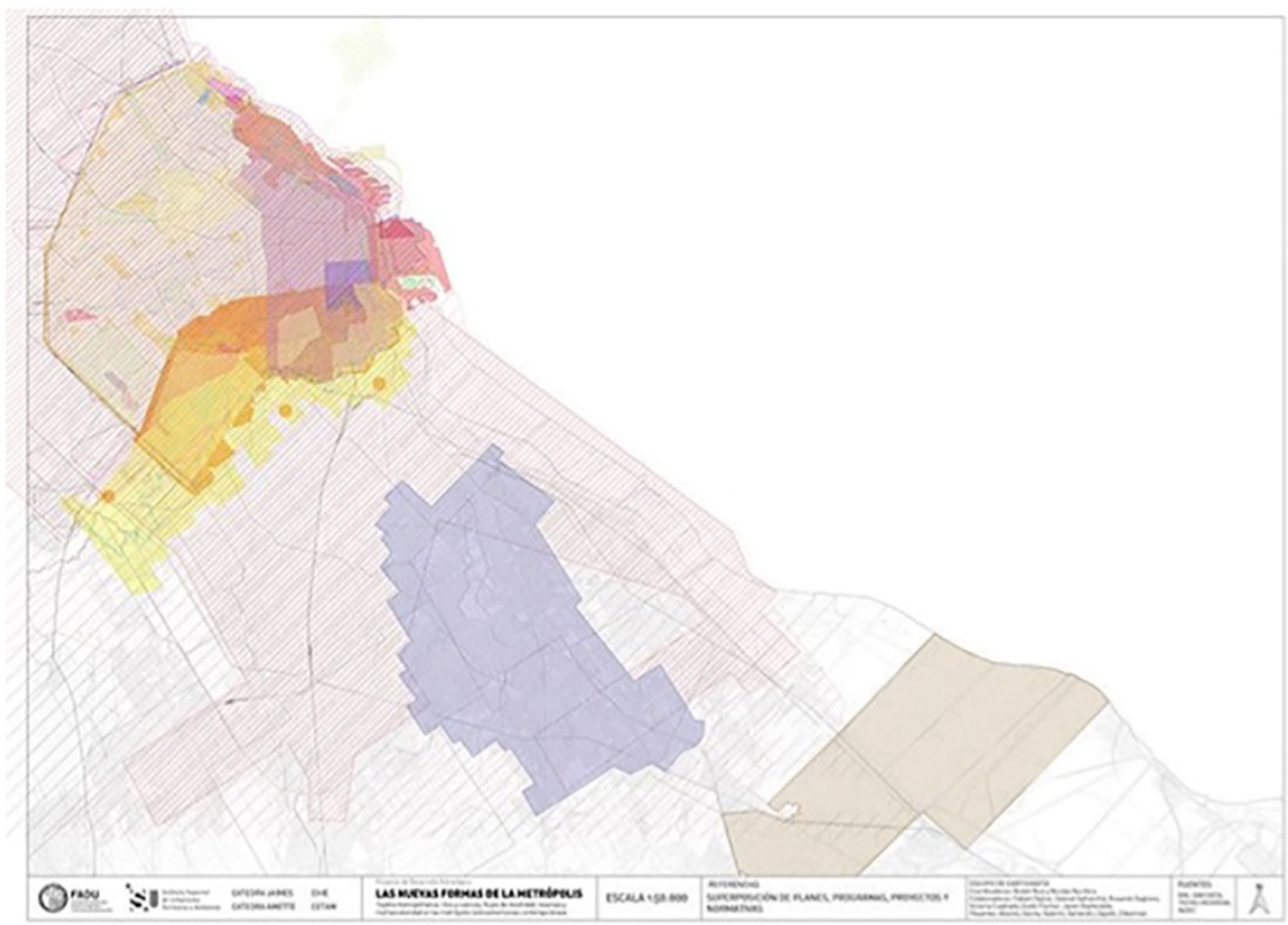

Figura 11. Región Metropolitana de Buenos Aires. Superposición de instrumentos urbanísticos metropolitanos entre 1880 y 2020 . Escala 3 (1:50.000). Área metropolitana de 2.500 $\mathrm{km}^{2}$. Fuente: Proyecto de Desarrollo Estratégico (PDE) Las Nuevas Formas de la Metrópolis, Universidad de Buenos Aires / Instituto Superior de Urbanismo, Territorio y Ambiente (2018) 


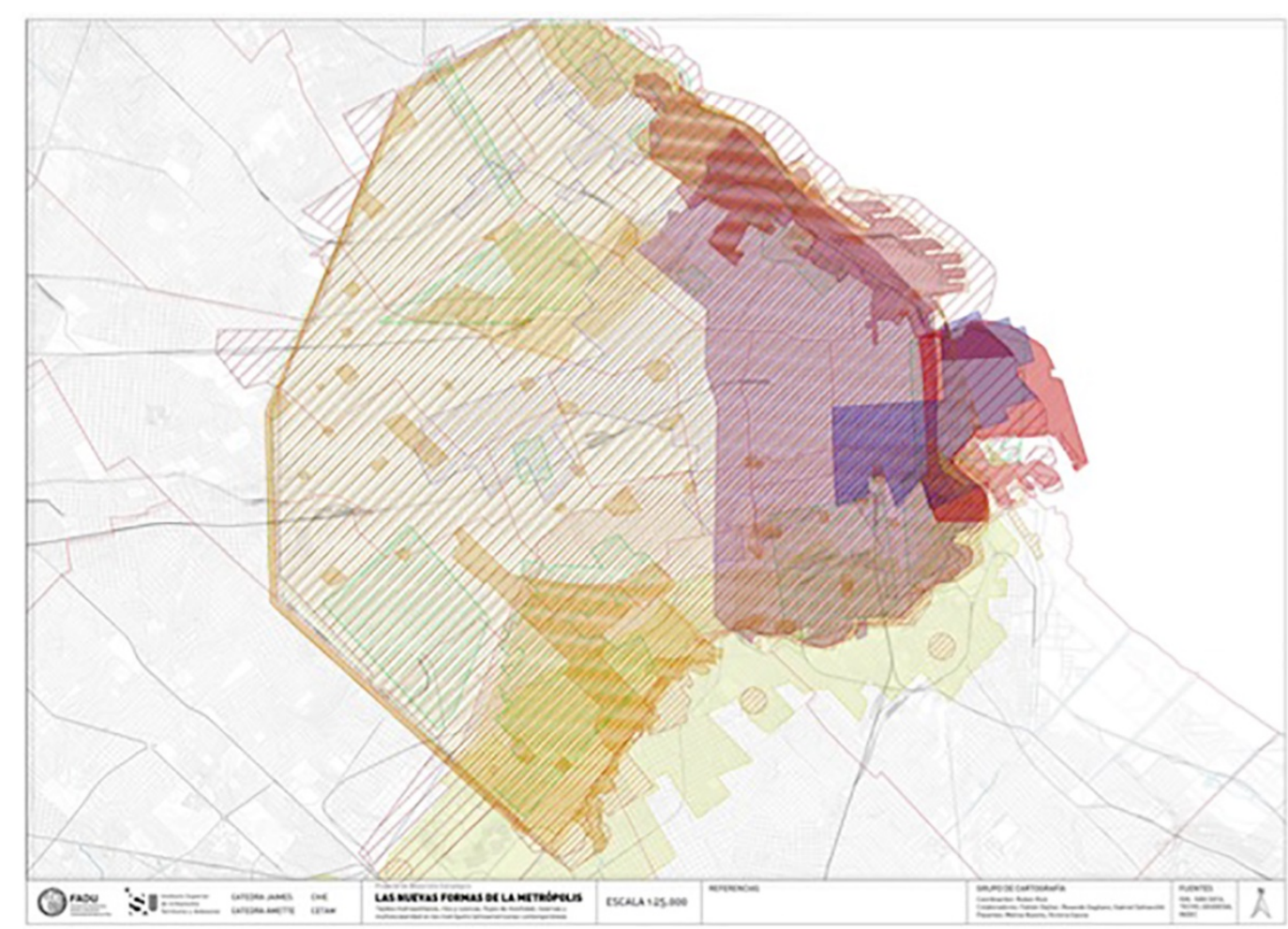

Figura 12. Región Metropolitana de Buenos Aires. Superposición de instrumentos urbanísticos metropolitanos entre 1880 y 2020 . Escala 4 (1:25.000). Área urbana de $625 \mathrm{~km}^{2}$. Fuente: Proyecto de Desarrollo Estratégico (PDE) Las Nuevas Formas de la Metrópolis, Universidad de Buenos Aires / Instituto Superior de Urbanismo, Territorio y Ambiente (2018)

4.2.2. Trabajo con Adoptantes: recopilación de planes, proyectos, programas y regulaciones para su nivel jurisdiccional, según el orden escalar de estudio.

Definido el orden escalar de estudio, una de las tareas fue preparar las bases para el trabajo con los Adoptantes a realizar durante el segundo año del proyecto.

Así, el trabajo con la Corporación Buenos Aires Sur Sociedad del Estado ha identificado los instrumentos desarrollados en su marco, cuyas siluetas se han asimismo redibujado y transferido a la cartografía en el orden escalar definido. 


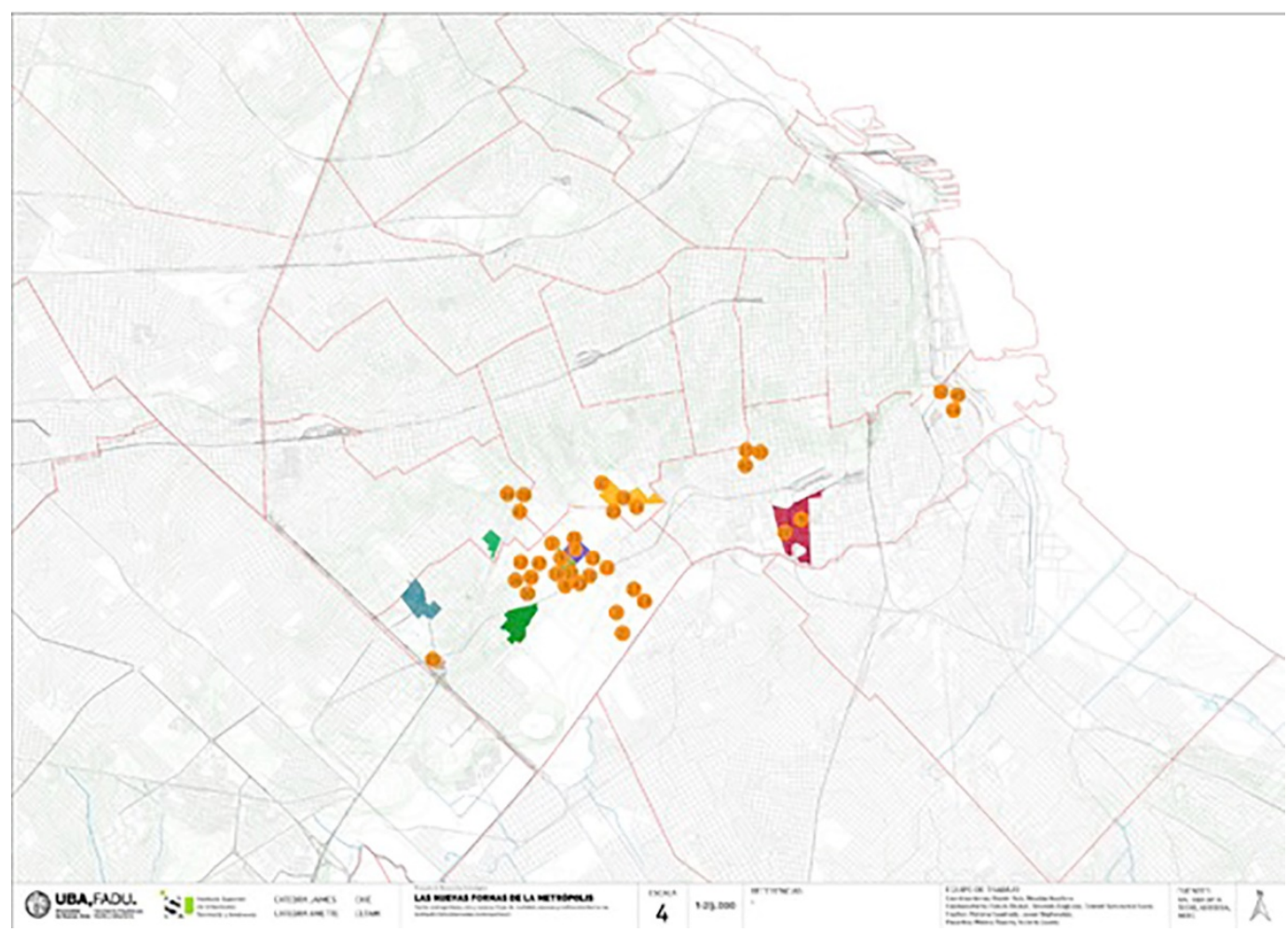

Figura 13. Corporación Buenos Aires Sur Sociedad del Estado. Superposición de instrumentos urbanos entre 2008 y 2020 . Escala 4 (1:25.000). Área urbana de $625 \mathrm{~km}^{2}$. Fuente: Proyecto de Desarrollo Estratégico (PDE) Las Nuevas Formas de la Metrópolis, Universidad de Buenos Aires / Instituto Superior de Urbanismo, Territorio y Ambiente (2018)

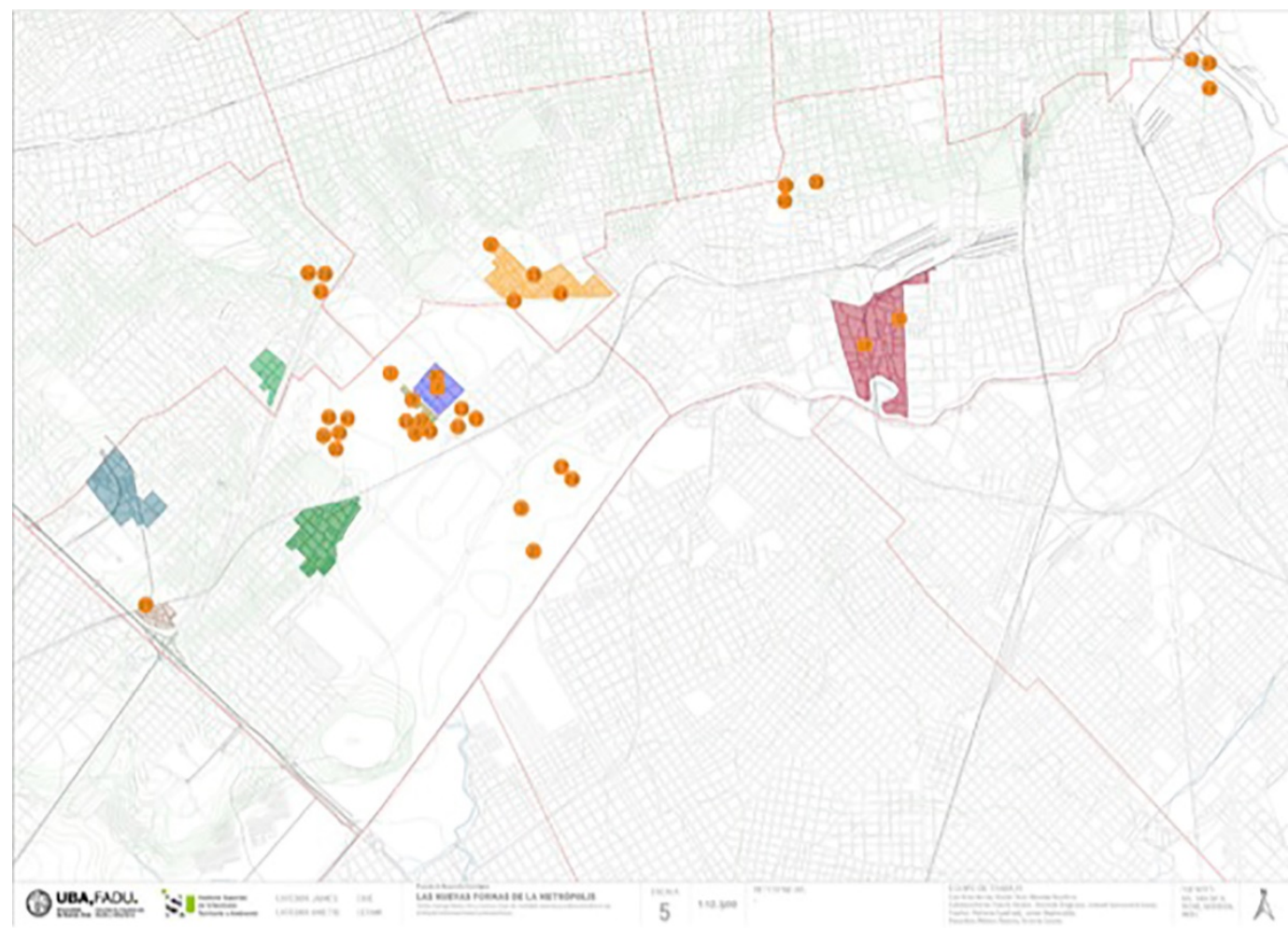

Figura 14. Corporación Buenos Aires Sur Sociedad del Estado. Superposición de instrumentos urbanos entre 2008 y 2020 . Escala 5 (1:12.500). Área urbana de $150 \mathrm{~km}^{2}$. Fuente: Proyecto de Desarrollo Estratégico (PDE) Las Nuevas Formas de la Metrópolis, Universidad de Buenos Aires / Instituto Superior de Urbanismo, Territorio y Ambiente (2018) 


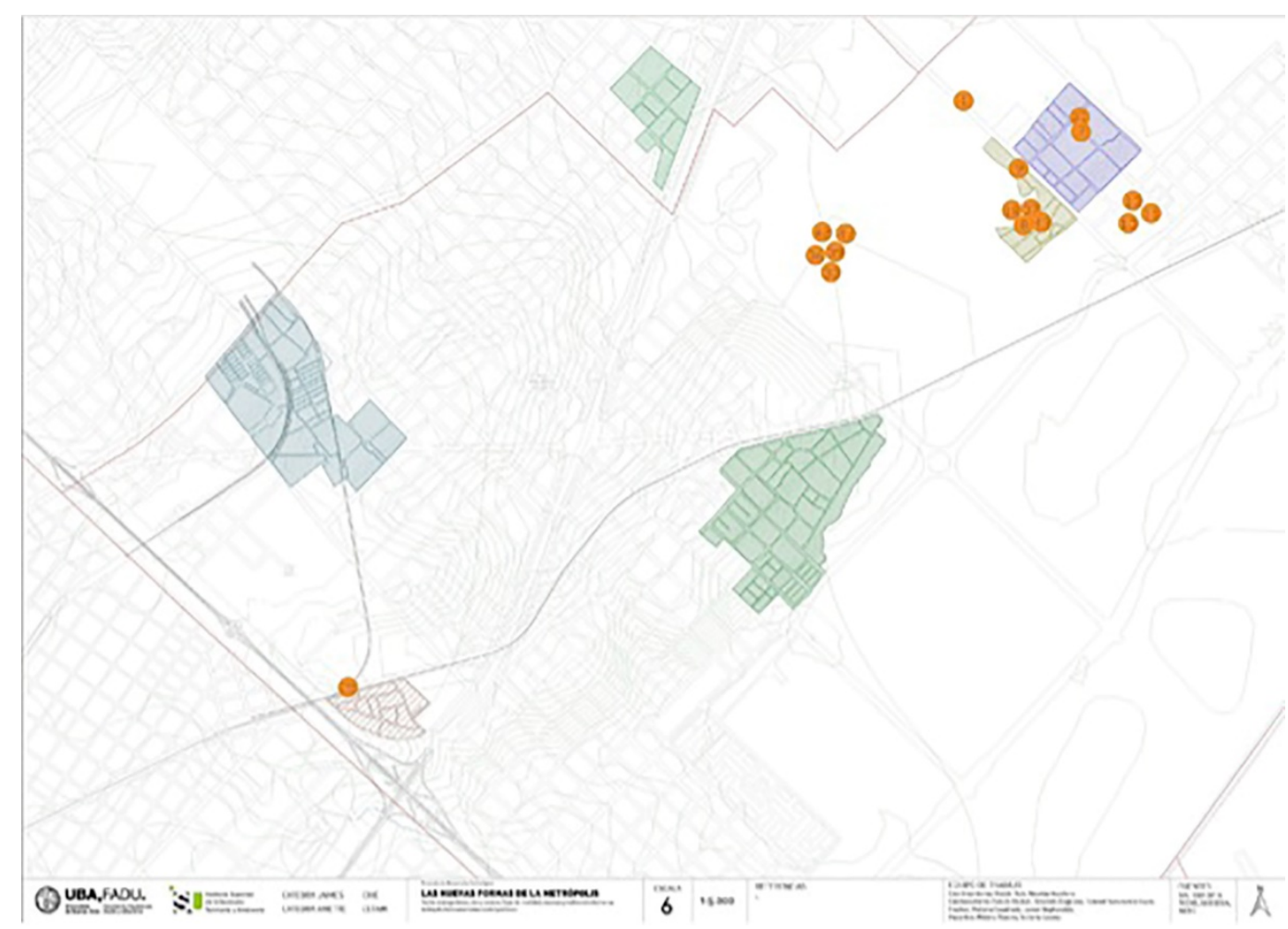

Figura 15. Corporación Buenos Aires Sur Sociedad del Estado. Superposición de instrumentos urbanos entre 2008 y 2020 . Escala 6 (1:5.000). Área urbana de $25 \mathrm{~km}^{2}$. Fuente: Proyecto de Desarrollo Estratégico (PDE) Las Nuevas Formas de la Metrópolis, Universidad de Buenos Aires / Instituto Superior de Urbanismo, Territorio y Ambiente (2018)

4.2.3. Recopilación de una selección de planes, proyectos, programas y regulaciones de vivienda en la Región Metropolitana de Buenos Aires.

A efectos de alcanzar los órdenes escalares menores del proyecto, y por su relevancia durante el Siglo XX para la reconfiguración del tejido metropolitano, una de las tareas principales de los estudiantes de grado que realizan sus pasantías de formación en investigación fue reunir y redibujar bajo códigos comunes una selección de planes, proyectos, programas y regulaciones de vivienda promovidos por el Estado durante el citado siglo. 


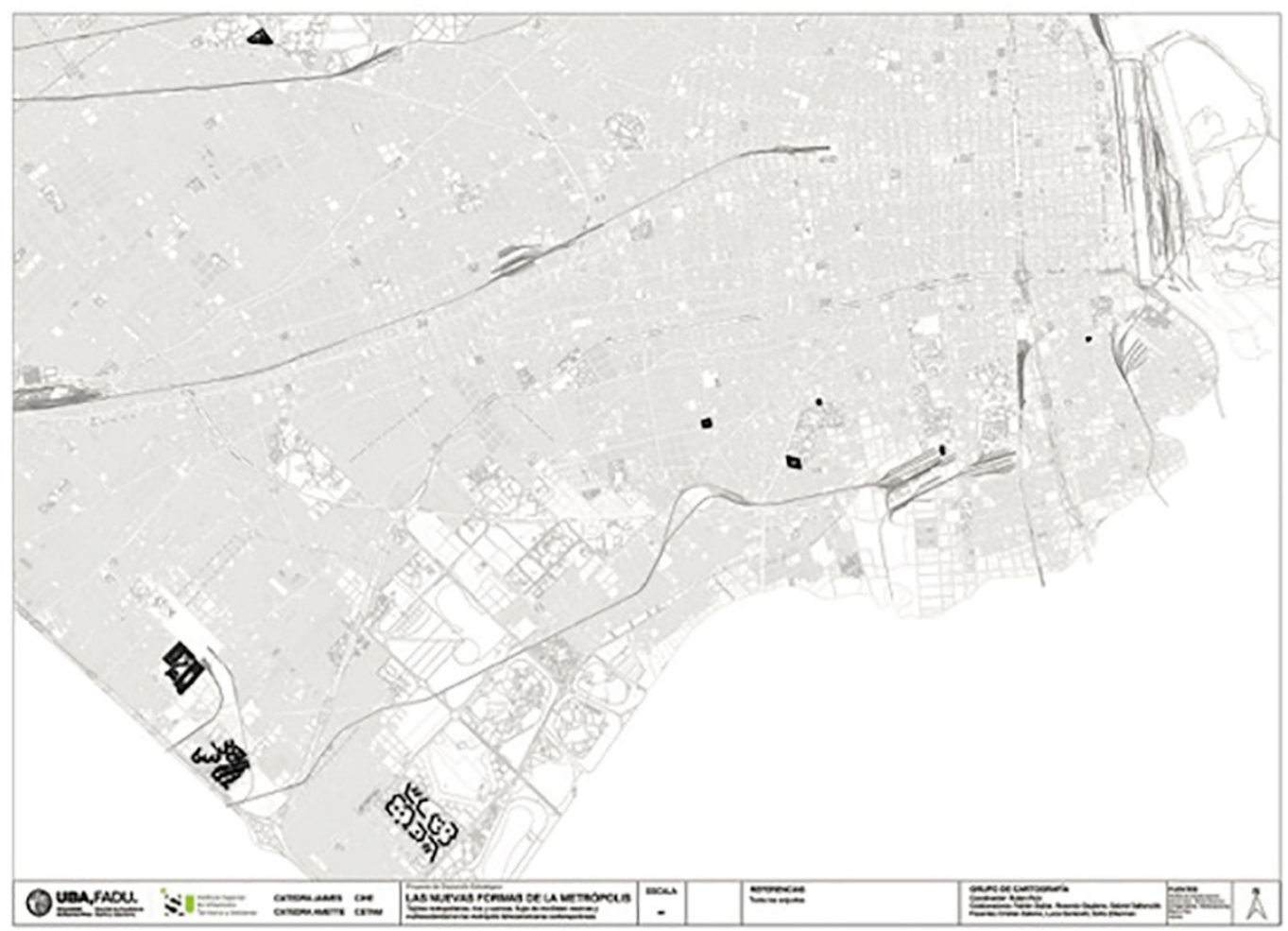

Figura 16. Recopilación de una selección de planes, proyectos, programas y regulaciones de vivienda en la Región Metropolitana de Buenos Aires. Escala 5 (1:12.500). Área urbana de 150km². Fuente: Proyecto de Desarrollo Estratégico (PDE) Las Nuevas Formas de la Metrópolis, Universidad de Buenos Aires / Instituto Superior de Urbanismo, Territorio y Ambiente (2018)

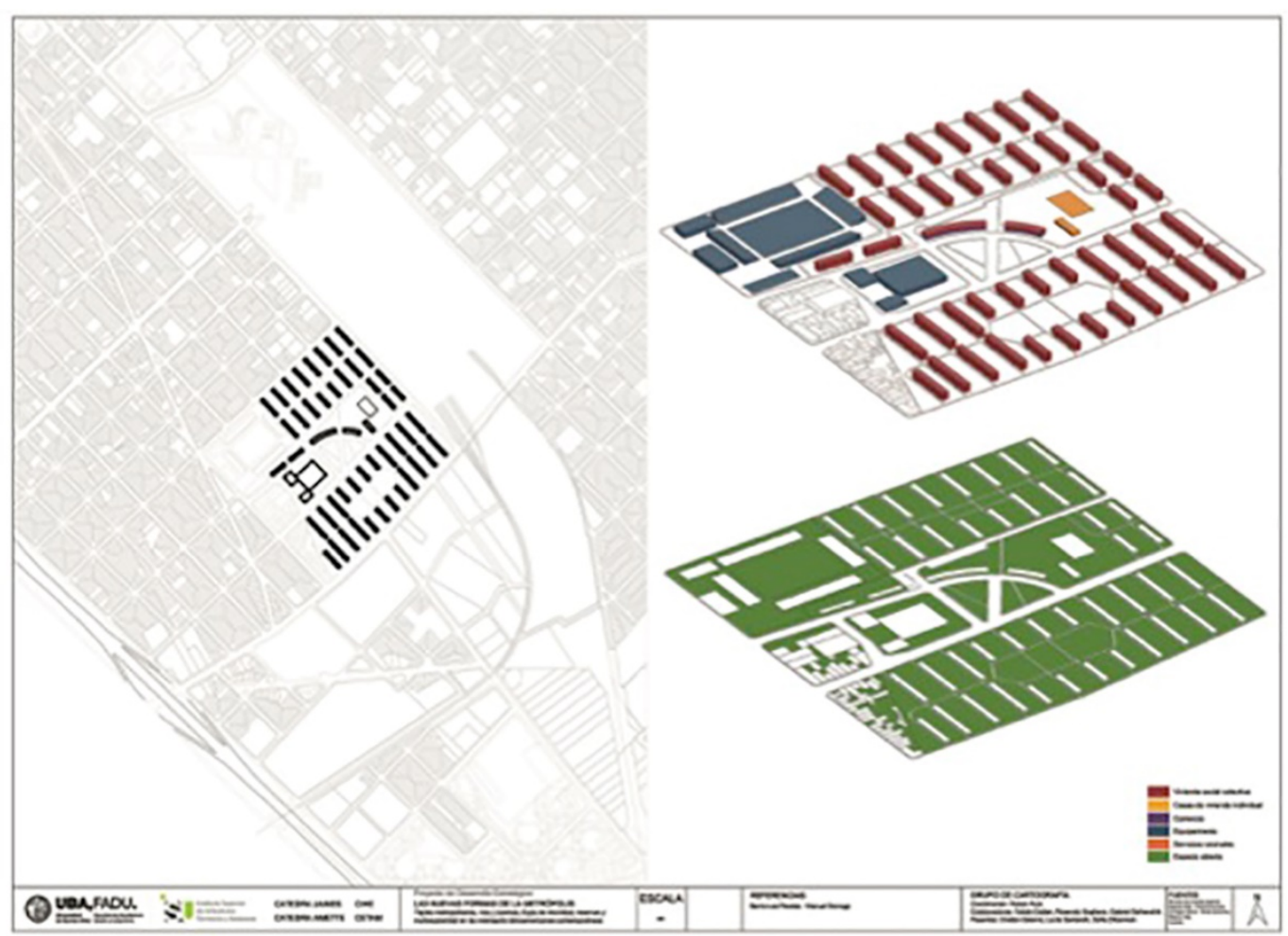

Figura 17. Recopilación de una selección de planes, proyectos, programas y regulaciones de vivienda en la Región Metropolitana de Buenos Aires. Barrio Los Perales, 1947-49. Escala 8 (1:1.000). Área urbana de 100Ha. Fuente: Proyecto de Desarrollo Estratégico (PDE) Las Nuevas Formas de la Metrópolis, Universidad de Buenos Aires / Instituto Superior de Urbanismo, Territorio y Ambiente (2018) 


\subsection{Recopilación de trabajos producidos en la Carrera de Grado de Arquitectura de la UBA-FADU, en las asignaturas de contenidos urbanos exclusivos.}

La producción académica durante la formación de arquitectos y urbanistas, en términos de reflexión y producción de una abundante cantidad de proyectos en el contexto de una enseñanza masiva (en la cual se enmarca el presente proyecto de investigación), da cuenta de intereses concurrentes respecto de la identificación de problemáticas urbano-metropolitanas, no sólo en términos de extensión y forma urbana, sino también sobre las dimensiones políticas, sociales, culturales, ambientales y otras diversas involucradas en todo fenómeno urbano.

Es por ello que se ha considerado relevante para el proyecto producir una recopilación de trabajos producidos en las asignaturas de contenidos urbanos exclusivos (Morfología Urbana, Planificación Urbana, Proyecto Urbano) de los últimos años, cuya sistematización en el segundo año de trabajo permitirá establecer una base para el trabajo comparativo, identificar áreas de superposición y vacancia, y una comparación con el plexo de instrumentos urbanísticos producidos por el Estado.

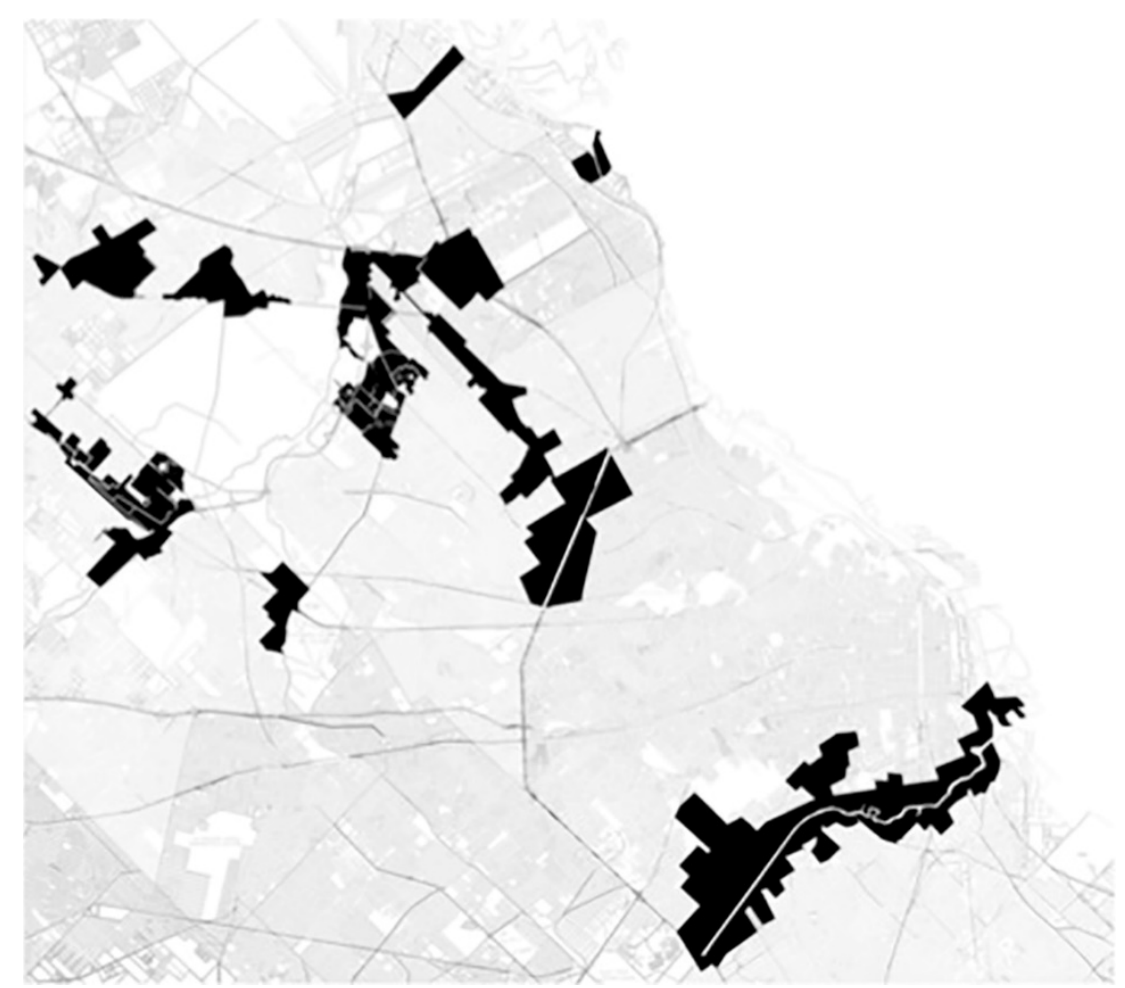

Figura 18. Región Metropolitana de Buenos Aires. Recopilación de trabajos producidos en la Carrera de Grado de Arquitectura de la UBAFADU, en las asignaturas de contenidos urbanos exclusivos. Escala 3 (1:50.000). Área metropolitana de $2.500 \mathrm{~km}^{2}$. Fuente: Proyecto de Desarrollo Estratégico (PDE) Las Nuevas Formas de la Metrópolis, Universidad de Buenos Aires / Instituto Superior de Urbanismo, Territorio y Ambiente (2018) 

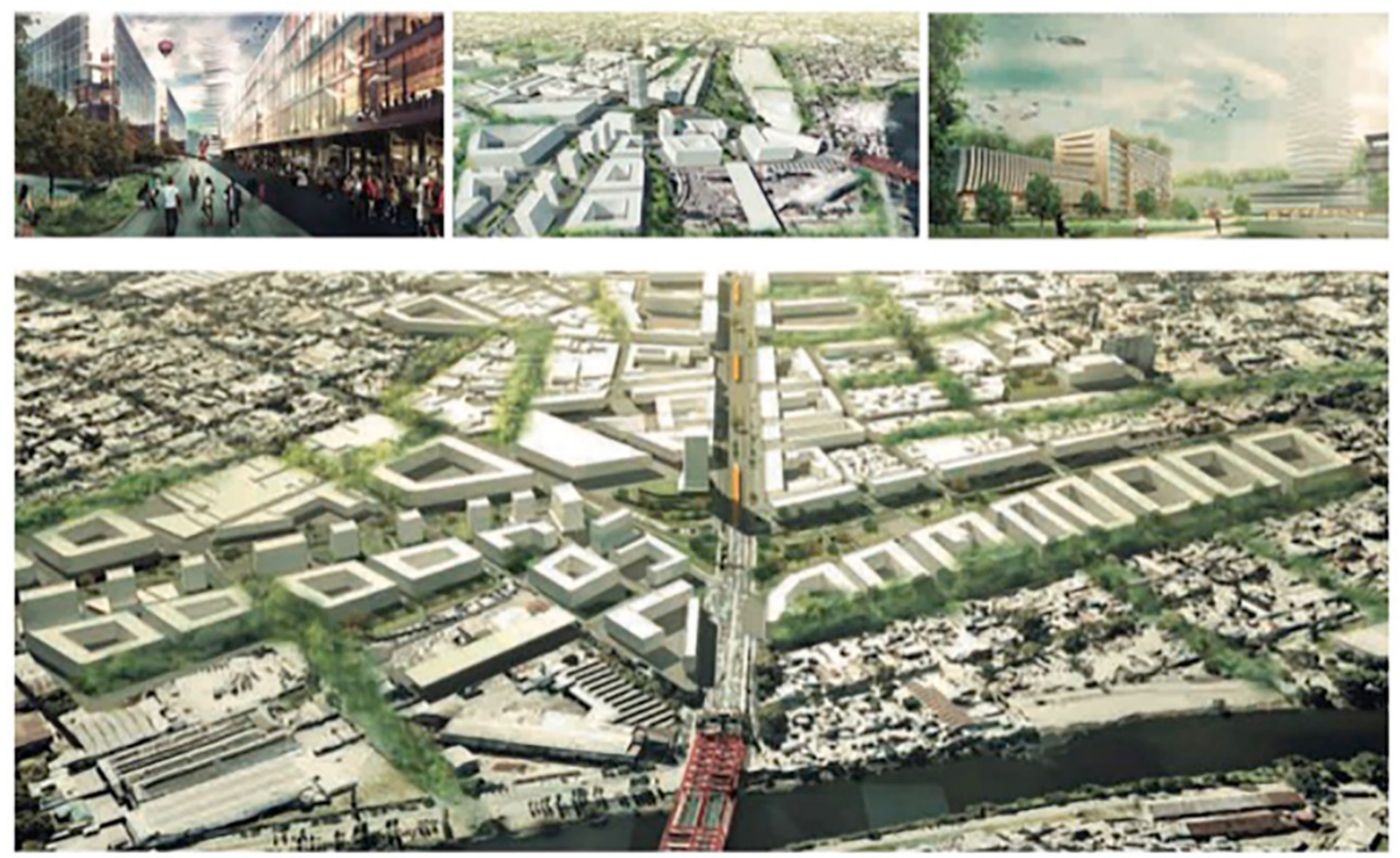

Figura 19. Región Metropolitana de Buenos Aires. Recopilación de trabajos producidos en la Carrera de Grado de Arquitectura de la UBA-FADU, en las asignaturas de contenidos urbanos exclusivos. Fuente: Proyecto de Desarrollo Estratégico (PDE) Las Nuevas Formas de la Metrópolis, Universidad de Buenos Aires / Instituto Superior de Urbanismo, Territorio y Ambiente (2018) 


\section{Las Nuevas Formas de la Metrópolis. Etapa 1 de la Investigación: Segunda Fase} Caracterización de tipologías urbanas y determinación de tendencias en el orden escalar definido.

La identificación de tipologías urbanas características de asentamiento, densificación, extensión y transformación en el sistema de la Región Metropolitana de Buenos Aires, en el orden escalar que se ha definido, requiere tanto para su producción y desarrollo, como para su transferencia, de dispositivos de comparación y comunicación eficaces.

Es así como, en el último tramo del trabajo del primer año, el proyecto ha desarrollado una clasificación sintética y comparativa en términos gráficos, que distingue en cinco colores otros tantos tipos de instrumentos urbanísticos metropolitanos (regulaciones, organismos, planes, programas, y proyectos) organizando en las ordenadas sus magnitudes en términos de área de suelo, y en las abscisas las décadas de su producción.

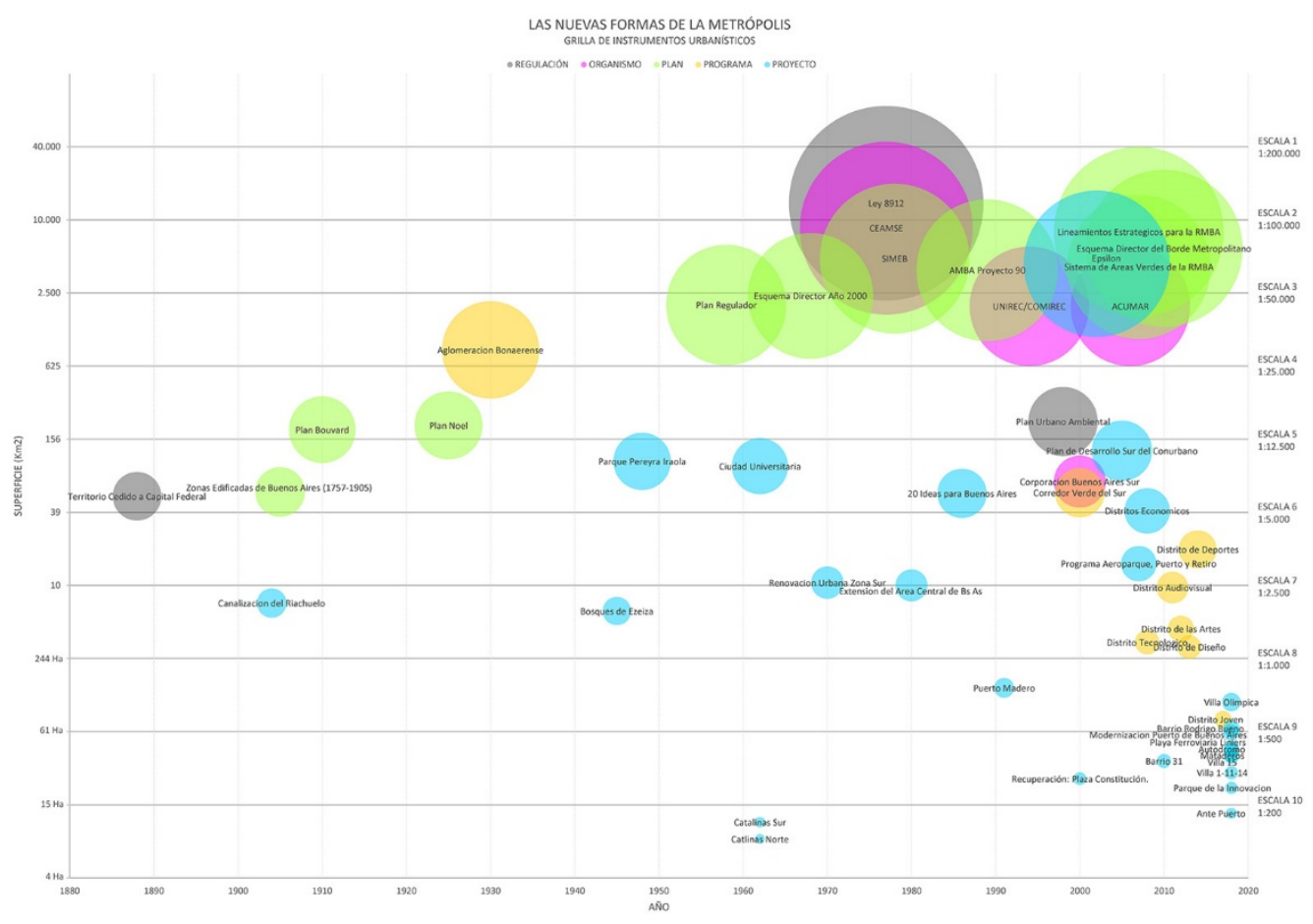

Figura 20. Región Metropolitana de Buenos Aires. Clasificación escalar de instrumentos urbanísticos metropolitanos entre 1880 y 2020 : regulaciones, organismos, planes, programas, y proyectos. Fuente: Proyecto de Desarrollo Estratégico (PDE) Las Nuevas Formas de la Metrópolis, Universidad de Buenos Aires / Instituto Superior de Urbanismo, Territorio y Ambiente (2018) 


\section{Comentarios, conclusiones preliminares y aperturas}

Explorar la mutua implicación entre las dimensiones de lo territorial, lo metropolitano, lo urbano y lo arquitectónico, abordando como caso de estudio y operación los tejidos genéricos de Buenos Aires, supone la recuperación y actualización de debates de las últimas décadas acerca de la validez de modelos urbanos históricamente caracterizados como instrumentos de inclusión, diversidad, y complejidad (Díaz, 2013; Pope, 2015), para abordar una agenda presente de transformaciones urbanas que tiende en buena medida a convertir a estos valores en sus opuestos.

Si bien de manera aún incipiente (Figura 20), el orden escalar espacial propuesto para la investigación va permitiendo identificar fijaciones o anclajes escalares en los que observar el despliegue de los instrumentos urbanísticos metropolitanos 1880-2020 relevados; habilitando para, durante el segundo año del proyecto, estudiar el comportamiento de la forma metropolitana-urbana (tanto la presente como que se propondrá) en términos de integración y segregación, inclusive como marco para la investigación de significaciones sociales y su despliegue temporal.

Asimismo, para los diferentes niveles de gobierno implicados en lo metropolitano, entre los que se encuentran ambos organismos adoptantes de los resultados del proyecto, resulta crucial avanzar en nuevos posibles de forma urbana, para contribuir a operar de modo consistente vis a vis las fuerzas que tienden a la fragmentación de esta forma y, como consecuencia, de la experiencia democrática de la ciudad.

El proyecto postula que es posible y necesario proveer desde la investigación basada en la noción de proyecto estos nuevos posibles, capaces ellos de actuar en el tenso campo de lo sociopolítico urbano que tiene lugar en el espacio de una ciudad contemporánea en conflicto. Este tipo de aproximación puede además agregar perspectivas a la literatura sobre lo escalar y la fragmentación en los estudios urbanos (Brenner, 1998, 2000, 2016), centrada en determinadas escalas de despliegue que no alcanzan todavía a postular, al mismo tiempo, de qué modo concreto las formas urbanas son capaces o no de colaborar, o de confrontar, o en cualquier caso de ponerse en relación, tanto con las políticas del Estado como con las fuerzas económicas en el espacio multiescalar metropolitano que definen. Las versiones de forma urbana que ofrece hoy nuestra cultura disciplinar proyectual no son escalares: bien se trata de inserciones en tejidos históricos de pequeña escala agregados y extendidos, bien de experiencias más o menos integradas o excluyentes de ciudad modernista, bien de proyectos urbanos entendidos como enclaves discretos en el continuo de la ciudad. Ninguna de estas versiones parece ser hoy suficientemente operativa.

Premisa para los instrumentos urbanísticos a proponer en la segunda etapa de la investigación es que ellos no actúen tanto como respuesta o reacción frente a situaciones escalares identificadas previamente, sino como instancias de comparación con estas situaciones; convirtiéndose en instrumentos estándar de diagnóstico y medida, capaces de ser empleados, en tanto tales, para interpretar y operar en áreas de la ciudad metropolitana sobre las cuales los instrumentos tradicionales, tal como se los viene entendiendo, cada vez menos tienen para ofrecer. 


\section{BIBLIOGRAFÍA}

ABBA, A. (2010) Metrópolis argentinas: agenda política, institucionalidad y gestión de las aglomeraciones urbanas interjurisdiccionales. Buenos Aires: Café de las Ciudades.

ABBA, A. (2014) Identificación de la estructura socioterritorial de la Ciudad de Buenos Aires teniendo en cuenta su contexto metropolitano. Informe Final. Buenos Aires: Facultad de Arquitectura, Diseño y Urbanismo (Observatorio Urbano Local - Buenos Aires Metropolitana, CIHaM) - Unidad de Proyectos Especiales Área Metropolitana de Buenos Aires (UPEAMBA). Inédito.

BRENNER, N. (1998) Between fixity and motion: accumulation, territorial organization and the historical geography of spatial scales. Environment and Planning D: Society and Space. Vol. 16 (4), pp. 459-481.

BRENNER, N. (2000) The urban question as a scale question: reflections on Henri Lefebvre, urban theory and the politics of scale. International Journal of Urban and Regional Research. Vol. 24 (2), pp. 361-378.

BRENNER, N. (2016) Critique of Urbanization: Selected Essays. Basilea: Birkhäuser.

DÍAZ, T. (2013) La arquitectura después de la Metrópolis. En: Plot. Vol. 16, pp. 180-186.

GARAY, A. (coord.) (2007) Lineamientos para la Región Metropolitana de Buenos Aires. Subsecretaría de Urbanismo y Vivienda, Ministerio de Infraestructura, Vivienda y Servicios Públicos, Provincia de Buenos Aires.

GÓMEZ GAVAZZO, Carlos (1973) De la Estética a la Economía. Montevideo: Instituto de Teoría de la Arquitectura y Urbanismo, Universidad de la República.

McCANN, E. J. (2003) Framing space and time in the city: urban policy and the politics of spatial and temporal scale. Journal of Urban Affairs. Vol. 25 (2), pp. 159-178.

POPE, A. ([1996] 2015) Ladders. New York: Princeton Architectural Press.

VECSLIR, L., y ELINBAUM, P. (2016) Centro y centralidades en la Región Metropolitana de Buenos Aires. En: Janches, F.; Amette, R.; Jaimes, C.; Corti, M. (comps.) (2016) Del Conocimiento al Desarrollo: Nuevos desafíos de la Universidad en el desarrollo urbano contemporáneo. Buenos Aires: Eudeba, pp. 420-438.

Fuentes electrónicas:

Observatorio Metropolitano. Consejo Profesional de Arquitectura y Urbanismo, Buenos Aires, Argentina. http://www.observatorioamba.org (Consulta: 2018).

Decreto 1126/2016 de creación de la CO.C.A.M.B.A.. Ministerio del Interior, Obras Públicas y Vivienda, República Argentina, 26/10/2016.

htttps://www.boletinoficial.com.ar/pdf/linkQR/WGZKYjhFbUpTRkOrdTVReEh2ZkU0dz09 